\title{
Forcing and the Universe of Sets: Must we lose insight?
}

\author{
Neil Barton*
}

29 January $2018^{\dagger}$

\begin{abstract}
A central area of current philosophical debate in the foundations of mathematics concerns whether or not there is a single, maximal, universe of set theory. Universists maintain that there is such a universe, while Multiversists argue that there are many universes, no one of which is ontologically privileged. Often forcing constructions that add subsets to models are cited as evidence in favour of the latter. This paper informs this debate by analysing ways the Universist might interpret this discourse that seems to necessitate the addition of subsets to $V$. We argue that despite the prima facie incoherence of such talk for the Universist, she nonetheless has reason to try and provide interpretation of this discourse. We analyse extant interpretations of such talk, and argue that while tradeoffs in naturality have to be made, they are not too severe.
\end{abstract}

\section{Introduction}

Recent discussions of the philosophy of set theory have often focussed on how many universes of sets there are. The following is a standard position:

Universism. There is a unique, maximal, proper class sized universe containing all the sets (denoted by ' $V$ ').

Universism has often been thought of as the 'default' position on the ontology of sets ${ }^{1}$ However, some have seen the existence of many different epistemic possibilities for the nature of the set-theoretic universe, shown by the large diversity of model-theoretic constructions witnessing independence (we discuss two of these methods later) as indicative of the existence of a diversity of different set-theoretic universes. In this paper, we will be concerned with forcing. This technique has been undeniably central in the study of independence in set theory, and (prima facie at

\footnotetext{
*Kurt Gödel Research Center for Mathematical Logic (KGRC), Währinger Straße, 25, 1090, Vienna, Austria, E-mail: neil.barton@univie.ac.at.

†The author wishes to thank Carolin Antos, Andrés Eduardo Caicedo, Monroe Eskew, Sy Friedman, Vera Flocke, Victoria Gitman, Bob Hale, Joel Hamkins, Toby Meadows, Alex Paseau, Ian Rumfitt, Chris Scambler, Jonathan Schilhan, Daniel Soukup, Kameryn Williams, and two anonymous reviewers from The Review of Symbolic Logic for insightful and helpful comments, as well as audiences in Cambridge, Vienna, and Toulouse for the opportunity to present and subsequent discussion. He is also very grateful for the generous support of the UK Arts and Humanities Research Council and FWF (Austrian Science Fund, Project P 28420).

${ }^{1}$ Both Gödel and Cantor, for example, are notable proponents.
} 
least) adds a set (or-as we shall see-a class) $G$ to a model $\mathfrak{M}$ to form a forcing extension extension $\mathfrak{M}[G]$.

In broad strokes, the problem arises when we note that set-theoretic practice is replete with uses of forcing where the symbol ' $V$ ' is used to denote the model over which we force (the so-called 'ground model'). Since, for non-trivial forcing, the relevant $G$ added cannot be in the ground model, one might naively think that the relevant $G$ lives outside $V$, and so the Universist position is immediately false.

As is well-known, however, there are various interpretative strategies available to the Universist when it comes to forcing. We discuss these in detail later, but for now we note that two popular options have been the use of countable transitive models and Boolean-valued models. In these cases, we re-interpret some or all of the terms in the forcing construction to yield an interpretation of the forcing talk that does not commit us to sets outside $V$. Opponents of Universism sometimes argue that these interpretations are problematic ${ }^{2}$ In this paper, we argue for the following claims:

(1.) There is some pressure arising from set-theoretic practice to provide an interpretation of forcing where the use of ' $V$ ' in a forcing construction is linked to actual truth in $V$.

(2.) Nonetheless, the Universist has several options available, and only mild compromises have to be made for some of these interpretations.

In this way, we aim to advance and clarify the dialectic between the Universist and her opponents. In particular we will argue that underpinning (1.) are additional philosophical commitments to naturality of interpretation, and that these are (2.) largely satisfied when considering some extant possibilities for interpreting forcing constructions.

Here's the plan: After these introductory remarks, we ( $\$ 1)$ briefly outline the kinds of forcing construction we will consider. Next (§2) we will argue that despite the fact that the Universist has methods for interpreting the forcing required for independence results, there is nonetheless some pressure to interpret forcing in such a way that its use is linked to the use of the symbol ' $V$ ' in forcing constructions. Specifically, we will argue that the Universist is able (through the formulation of axioms and proving of theorems) to learn more about the structure of $V$ by viewing $V$ as situated in a multiverse framework. We'll argue then that if the Universist accepts certain constraints on the naturalness of interpretation of mathematical discourse, there is additional pressure on her interpretation of forcing. Finally (§3) we provide an analysis of extant interpretations of forcing found in the literature (the forcing relation, Boolean-valued models, the Boolean ultrapower, and countable transitive models). We argue that use of the forcing relation and Boolean-valued models largely violate the constraints of $\S 2$. We will argue that the situation for the Boolean ultrapower and countable transitive models is more subtle. While there are tradeoffs to be made for each interpretation, for the countable transitive model strategy these are relatively minimal, and for a certain class of forcing constructions the Boolean ultrapower is especially natural (and especially unnatural for a different class). We conclude with some open questions concerning the directions of debate for both the Universist and her critics.

\footnotetext{
${ }^{2} \mathrm{~A}$ good example here is [Hamkins, 2012].
} 


\section{Varieties of forcing}

We start with a brief description of forcing constructions to make plain some of their mathematical properties that will be philosophically relevant later. Forcing comes in two main kinds; set forcing and class forcing ${ }^{3}$ We briefly sketch the constructions (deferring to the relevant technical literature where appropriate) noting their similarities and differences. The details will be familiar to specialists, but a feel for some of the properties of the constructions is necessary for seeing the challenges faced.

\subsection{Set forcing}

For set forcing we begin with a partial order with domain $P$, ordering $\leqslant p$, and maximal element $1_{\mathbb{P}}$, denoted by ' $\mathbb{P}=\left\langle P, \leqslant \mathbb{P}, 1_{\mathbb{P}}\right\rangle^{\prime}$, and have $\mathbb{P} \in \mathfrak{M}$ for some $\mathbf{Z F C}$ mode $]^{4}$ $\mathfrak{M}$. The relevant $p \in P$ are known as conditions and effectively operate by providing partial information about membership of the new object to be defined. We then, via a careful choice of names (known as 'P-names' $)^{5}$, and evaluation procedure ${ }^{6}$ add a filter $G$ on $\mathbb{P}$ that intersects all dense sets of $\mathbb{P}$ in $\mathfrak{M}$ to $\mathfrak{M}$. The end result is a model $\mathfrak{M}[G]$ that (i) satisfies ZFC, (ii) has exactly the same ordinal height as $\mathfrak{M}$, and (iii) is strictly larger than $\mathfrak{M}$ (in the sense that $\mathfrak{M} \subset \mathfrak{M}[G]$ ) $]^{7}$

Forcing is an especially interesting philosophical construction for a number of reasons. First, it is historically significant in that it has been used to settle many open questions (the most famous examples being the independence of $\mathrm{CH}$ and $\mathrm{AC}$ ). Second, it is of central importance in virtue of its ubiquity across modern set-theoretic mathematics; much of set theory concerns constructing one model from another using forcing arguments. However, especially philosophically interesting is that it keeps models standard ${ }^{8}$ Assuming that the ground model $\mathfrak{M}$ is transitive, well-founded, and satisfies $\mathbf{Z F C}$, the forcing extension $\mathfrak{M}[G]$ (i) has the same ordinals as $\mathfrak{M}$, (ii) satisfies ZFC, and (iii) is transitive and well-founded. The fact that forcing keeps the models standard is significant; generic extensions of a standard model of ZFC are also ZFC-satisfying cumulative hierarchies.

The issue concerning forcing and $V$ is, of course, that if we wish to perform a non-trivial forcing where ' $V$ ' denotes the Universist's $V$ as the ground model, the relevant generic $G$ must lie outside $V{ }^{9}$ But $V$ was meant to be all the sets there are, and so such a $G$ does not exist.

\footnotetext{
${ }^{3}$ There is also the related notion of arithmetic forcing used to study models of second-order arithmetic. Since we are interested primarily in models of set theory here, we set this aside.

${ }^{4} \mathrm{~A}$ brief remark is in order here: One does not always force over models of full $\mathbf{Z F C}$, and forcing over models of weaker theories is well studied. Indeed, in several mathematics texts (such as [Kunen, 2013]), the 'official' approach is to use the reflection theorem to obtain a model of 'enough' $\mathbf{Z F C}$ to conduct the independence proof. We defer consideration of this issue until later.

${ }^{5} \mathrm{~A} \mathbb{P}$-name is a relation $\tau$ such that $\forall\langle\sigma, p\rangle \in \tau[" \sigma$ is a $\mathbb{P}$-name" $\wedge p \in \mathbb{P}]$. In other words, $\tau$ is a collection of ordered pairs, where the first element of each pair is a $\mathbb{P}$-name and the second is some condition in $\mathbb{P}$ (the definition is not vacuous in virtue of the empty set trivially being a $\mathbb{P}$-name).

${ }^{6}$ We evaluate $\mathbb{P}$-names by letting the value of $\tau$ under $G$ (written 'val $(\tau, G)^{\prime}$ or ' $\tau_{G}$ ') be $\{\operatorname{val}(\sigma, G) \mid \exists p \in G(\langle\sigma, p\rangle \in \tau)\}$. The valuation operates stepwise by analysing the valuation of all the names in $\tau$ and then either adding them to $\tau_{G}$ (if there is a $p \in G$ and $\langle\sigma, p\rangle \in \tau$ ) or discarding them (if there is no such $p \in G$ ).

${ }^{7}$ It should be noted that in order for the forcing to be non-trivial, $\mathbb{P}$ has to be non-atomic (i.e. every $p \in P$ has incompatible extensions in $\mathbb{P}$ ).

${ }^{8} \mathrm{~A}$ model $\mathfrak{M}$ is normally called standard iff it has the real $\in$-relation. See [Kunen, 2013], §IV.2 for verification of the basic properties of forcing.

${ }^{9}$ This is because if $G$ were in $V, \mathbb{P}-G$ would be a dense set in $V$ missed by $G$.
} 


\subsection{Class forcing}

Class forcing is very similar to set forcing, except we drop the requirement that the partial order and generics are members of $\mathfrak{M}$ and instead permit proper-class-sized partial orders. The technique also uses partial orders with maximal elements $\left\langle\mathbb{P}, \leqslant_{\mathbb{P}}, 1_{\mathbb{P}}\right\rangle$, and adds a generic $G$ to our ground model $\mathfrak{M}$. The difference with class forcing is that $\mathbb{P}$ (and hence any associated dense classes and $G$ ) can now be proper-class-sized rather than just set-sized, and so while $\mathbb{P} \subset \mathfrak{M}$, it is not in general true that $\mathbb{P} \in \mathfrak{M}$, and $G$ is added as a class (from which it may be possible to construct new sets). The construction goes through largely the same as set forcing, with a few additional intricacies and features 10

Class forcing has some interesting properties not enjoyed by standard set forcing. A good example is that if we allow class forcing over $L$, then there are reals that we can add using class forcing that cannot be added by set forcings ${ }^{11}$ Further, using class forcing we can produce models that violate ZFC. For example, the partially ordered class $\operatorname{Col}(\omega, O n)$ (i.e. functions $p$ from finite subsets of $\omega$ into $O n$ ordered by reverse inclusion) is (without further constraints) perfectly legitimate. But if we force with this poset we obtain a model $\mathfrak{M}[G]$ that satisfies ZFC as long as $G$ is not allowed as a class predicate, as the first-order domains of $\mathfrak{M}$ and $\mathfrak{M}[G]$ are identical ${ }^{12}$ However, if we admit $G$ as a predicate into the language, Replacement fails. This is because $G$ codes a cofinal sequence from $\omega$ to $O n^{\mathfrak{M}[G]}$ and there is no set in $\mathfrak{M}[G]$ corresponding to $O n^{\mathfrak{M}[G]}{ }^{13}$ This is unlike the case of set forcing where $\mathfrak{M}[G]$ is guaranteed to satisfy $\mathbf{Z F C}$ if $\mathfrak{M}$ does. If $\mathbf{Z F C}$ preservation is desired ${ }^{14}$ some care ${ }^{15}$ is required in defining the relevant $\mathbb{P} \subseteq \mathfrak{M}$ to be used in forcing.

Class forcing thus introduces two additional challenges not posed by set forcing. Whilst we must provide an interpretation of any sets that get added, we must also explain what happens with a forcing that violates ZFC, and also how we should understand the addition of a class without the addition of any sets that could underpin the 'change' in classes ${ }^{16}$

\footnotetext{
${ }^{10}$ Details of the presentation given here are available in [Friedman, 2000|, Chapter 2. When performing a class forcing, we generally force over models of the form $L(A)=\bigcup\left\{L\left(A \cap V_{\alpha}\right) \mid \alpha \in O n\right\}$. Any model $(M, A)$ of $\mathbf{Z F}$ (where we include Replacement for formulas mentioning $A$ ) can be changed to a model of this form by expanding it to a model $\left(M, A^{*}\right)$ where $A^{*}=\{\langle 0, x\rangle \mid x \in A\} \cup\left\{\left\langle 1, V_{\alpha}^{M}\right\rangle \mid \alpha \in O n^{M}\right\}$.

${ }^{11}$ This is a deep result of Jensen, see [Friedman, 2010], p. 559 for details.

${ }^{12}$ To see this, note that for any $\mathbb{P}$-name $\sigma$ for this poset and for each condition $p$ in the intersection of the transitive closure of $\sigma$ with $\mathbb{P}, \operatorname{ran}(p) \subseteq \operatorname{rank}(\sigma)$. We then define the dense set $D=\{p \in \mathbb{P} \mid \operatorname{rank}(\sigma) \in \operatorname{ran}(p)\} . \quad D$ is then both dense and definable over $\mathfrak{M}$. Letting $\sigma^{p}=\left\{\tau^{p} \mid \exists q \in \mathbb{P}[\tau, q \in \sigma \wedge p \leqslant \mathbb{P} q]\right\}$. We then have $\sigma^{p}=\sigma^{G} \in M$ whenever $G$ is $\mathbb{P}$-generic over $\mathfrak{M}$ and $p \in D \cap G$, because $p$ either extends or is incompatible with any condition in the transitive closure of $\sigma$. Hence, whenever $G$ is $\mathbb{P}$-generic over $\mathfrak{M}$, they contain exactly the same first-order objects.

${ }^{13}$ For details, see [Holy et al., 2016].

${ }^{14}$ We shall argue later that for the purposes of talking about forcings over $V$, there is no reason why ZFC preservation is especially desirable.

${ }^{15}$ The relevant conditions are pretameness and tameness of the partial order, corresponding respectively to preservation of Replacement and Power Set. See [Friedman, 2000] for details.

${ }^{16} \mathrm{~A}$ delicate issue here is exactly what theory our starting universe satisfies when performing a class forcing. |Friedman, 2000| is a thorough presentation, and studies class forcing over structures of the form $(M, A)$, where $A$ is first-order definable with parameters. However, we might also try to force over models of full Morse-Kelley class theory, see Antos and Friedman, F] for discussion. Since our arguments here do not depend on the use of non-definable classes and, as it stands, the definable class presentation is more usual, we restrict our attention to constructions defined within this latter approach (though our arguments could easily be transferred to the MK case).
} 


\section{Looking at $V$ through the Multiversist lens}

We thus have two kinds of construction that seem to add external entities to models, and the datum that often set theorists use the terms ' $V$ ' and ' $V[G]^{\prime}$ ' when performing a forcing construction. However, more must be done philosophically to create a genuine problem here. For example, why (given the Universist's 'predicament) can't she just insist that the use of the terms ' $V$ ' and ' $V[G]^{\prime}$ ' should not be understood literally, or that forcing is simply not a legitimate construction? In this section we'll provide some support to the idea that there is a genuine problem to be dealt with here, and one way of motivating this problem is via constraints on the naturality of interpretation.

We will simply take it as read that the Universist should not outright reject forcing as a legitimate mathematical technique. Forcing is an essential part of the working set theorist's toolkit, and clearly represents a coherent body of study. We are also moved by the more open-ended desire to provide the Universist's opponent with the tools she requires to construct her objection, rather than just rejecting the grounds for the question outright.

However, the idea that we should re-interpret the problematic terms is pertinent, and indeed the extent to which such a strategy can be carried out satisfactorily forms the focus of this paper. For now, we simply note that the Universist can provide interpretations of forcing that are non-vacuous. We discuss these approaches in detail $\S 3$, but two standard approaches are either to regard forcing statements as a façon de parler for speaking about Boolean-valued models or as concerned with countable transitive models of (a fragment of) ZFC ${ }^{17}$ Each method of interpretation, however, yields a model that does not commit the Universist to sets outside $V{ }^{18}$ This is pertinent, since one important use of forcing constructions has been to show independence results. Specifically, given a statement $\phi$ (such as the continuum hypothesis) we use forcing to construct a model of (a fragment of) ZFC $+\neg \phi$, thereby showing that $\phi$ is not a consequence of (a fragment of) ZFC (or some extension thereof). If all we wish to do is show essentially number-theoretic facts about relative provability, then there is little pressure to accept that forcing requires anything more than a non-vacuous interpretation; all we need to do is show that there is some model or other that can serve as the interpretation of the forcing language and as a counterexample to the claim that $\mathbf{Z F C} \vdash \phi$, and the Universist can already do this.

In order for the existence of forcing to provide evidence against the Universist then, more needs to be said. In the rest of this section we will develop this problem. We will argue for the following claims:

\footnotetext{
${ }^{17}$ Indeed, this is the strategy of many set theory textbooks. For example the Boolean-valued model strategy is put forward by |Jech, 2002]:

"As the properties of the generic extension can be described entirely withing the ground model, statements about $V[G]$ can be understood as statements in the ground model using the language of forcing." (|Jech, 2002], p. 201)

Whereas the countable transitive model strategy is the main strategy pursued in [Kunen, 2013] (see Ch. $4 \S 5)$.

${ }^{18}$ Koellner, for example, is sensitive to this point:

"The advocate of the universe view is unmoved by these considerations [arising from forcing] since the models produced are not candidates for the universe of sets, the first because it is an object within the universe of sets, the second because it is a description of a class-size structure which is not even of the relevant type. The mere existence of the model-theory of forcing (something that is uncontroversially accepted by both parties) is not sufficient to secure the multiverse conception. Something more needs to be said." (|Koellner, 2013], pp. 18-19)
} 
(i) Forcing is more than just a tool for proving number-theoretic consistency facts, it is also useful for formulating axioms and proving theorems about large infinitary objects in $V$.

(ii) Given an acceptance of the claim that providing a 'natural' or 'transparent' interpretation of mathematical discourse is desirable, the Universist has additional constraints on her interpretation of forcing.

\subsection{Proving theorems and formulating axioms}

How is forcing useful for more than proving number-theoretic facts? As we'll see, forcing over $V$ can be used in analysing the structure of large infinitary objects outside the domain of number theory. This is both with respect to proving theorems from within ZFC, but also for formulating axioms that go beyond ZFC (and proving theorems on their basis). As we'll discuss below, this puts some pressure on the claim that we might want more than just any old interpretation of the mathematicssince viewing $V$ as though it were part of a multiversist framework facilitates insight concerning $V$, we might desire an interpretation of this talk that respects as much intuitive content of the mathematics as possible. Before we provide this philosophical analysis though, we provide a brief description of the mathematical landscape to show the importance of this way of thinking.

\section{Proving theorems}

Within ZFC, there are a wide number of questions concerning $V$ that can be settled on the basis of considering forcing extensions. The rough strategy of such theorems is to show that if $V$ has a forcing extension such that $\phi$ (for some particular $\phi$ ) then some other sentence $\psi$ holds of $V$ (say by using absoluteness facts). For example ${ }^{19}$

Theorem 1. [Baumgartner and Hajnal, 1973] $\omega_{1} \longrightarrow(\alpha)_{n}^{2}$ for all finite $n$ and countable $\alpha$ (i.e. For all finite $n$ and countable $\alpha$, every partition of the two-element subsets of $\omega_{1}$ into a finite number of pieces has a homogeneous ${ }^{20}$ set of order-type $\alpha$ ).

The proof proceeds by finding a homogeneous set in a forcing extension where MA holds. This establishes that a certain tree from the ground model is non-wellfounded in the extension. We then know, by the absoluteness of well-foundedness, that the tree is also non-well-founded in the ground model, establishing the theorem.

The theorem is broadly illustrative of how one can use the perspective of extensions to prove facts about the ground model. One moves to an extension where one has ensured the existence of objects of a certain desirable kind, used these to reason about objects in the ground model, and then inferred via absoluteness facts (e.g. Lévy-Shoenfield absoluteness) that the ground model must in fact have the relevant properties. Importantly here, theorems like the above are not straightforwardly about number theory and the possibility of proof, but rather concern genuine infinitary objects in the ground model.

A compendium of similar theorems is available in [Todorčević and Farah, 1995]. One further clear example bears mentioning here though. Since the formulation of $\mathrm{CH}$ (and realisation that it is independent from $\mathbf{Z F C}$ ) set theorists have studied so-called cardinal characteristics; the study of uncountable infinite sets that may or

\footnotetext{
${ }^{19} \mathrm{I}$ am grateful to [name removed for blind review] for pointing out this example.

${ }^{20}$ Here, a homogeneous set is a subset $X$ of $\omega_{1}$ such that every 2-element subset of $X$ is in the same member of the partition.
} 
may not be smaller than the continuum. The relationships of these infinite cardinals are well studied ${ }^{21}$, but recently [Malliaris and Shelah, 2016] showed that two such characteristics (namely $\mathfrak{p}$ and $\mathfrak{t}$ ) that were previously thought to be likely separable in certain models were in fact equal. The proof is rather involved 2 , but depends crucially on supposing for contradiction that $\mathfrak{p}<\mathfrak{t}$ in $V$, and then tracing out some consequences of this assumption (and finding a contradiction) in a forcing extension $V[G]$. Thus, situating $V$ within a Forcing Multiversist framework allows us to solve importan ${ }^{23}$ and difficult set-theoretic questions on the basis of how $V$ behaves with respect to its forcing extensions.

Thus, forcing extensions can function with respect to $V$ in contemporary set theory somewhat like the historical situation with complex and real numbers. For several years, before the advent of interpretation of the complex numbers in the Euclidean plane, mathematicians were still using the algebraic properties of complex numbers to prove results about the reals with great success ${ }^{24}$ Similarly, extensions of $V$ can be used to determine properties of $V$. It is the external perspective provided by the forcing extension that can facilitate a greater understanding of the ground model. The Universist then, might feel some pressure to interpret forcing over $V-$ by looking at $V$ from $V[G]$, she is able to see more.

\section{Formulating axioms}

The same is true also for theories extending ZFC. Especially interesting here is that forcing becomes a useful tool not just for proving theorems, but also formulating axioms. As we explain below, the flexibility afforded by extensions often provides us with additional resources for expressing axioms with interesting properties.

One way of asserting the existence of large cardinals (often quite strong), is through the use of elementary embeddings. The cardinals measurable, strong, supercompact (among others) are all naturally defined by positing the existence of elementary embeddings from $V$ into transitive inner models. These represent strong axioms, pushing us (unlike smaller large cardinals) well beyond $V=L$. When defining a large cardinal through an embedding $j: \mathfrak{N} \longrightarrow \mathfrak{M}$, the strength of the embedding depends mainly on two parameters:

(i) The size of $\mathfrak{N}$ and $\mathfrak{M}$.

(ii) Where $j$ sends the ordinals.

For instance, the minimal case for an embedding between proper class models; namely the existence of a non-trivial $j: L \longrightarrow L$ suffices to define the principle that " $0^{\sharp}$ exists". If we assume that $\operatorname{dom}(j)=V$, we strengthen to the level of a measurable cardinal. We know that the existence of a non-trivial $j: V \longrightarrow V$ is

\footnotetext{
${ }^{21}$ Chichoń's diagram, for example, is a manifestation of the detailed knowledge we now have concerning how some of these characteristics behave in models of ZFC.

${ }^{22}$ I thank [name removed for blind review] for patient explanation here.

${ }^{23}$ For instance, the result (unlike much of set theory) attracted some interest from the wider mathematical community, being the subject of a piece in Quanta magazine and receiving some attention from Tim Gowers.

${ }^{24}$ See, for example the remarks in [Painlevé, 1900] concerning real and complex analysis:

"The natural development of this work soon led the geometers in their studies to embrace imaginary as well as real values of the variable. The theory of Taylor series, that of elliptic functions, the vast field of Cauchy analysis, caused a burst of productivity derived from this generalization. It came to appear that, between two truths of the real domain, the easiest and shortest path quite often passes through the complex domain."
} 
inconsistent (modulo $\mathbf{Z F}$ ) with AC ${ }^{25}$ Despite this we can study intermediate cardinals by modifying the properties of $j$ and $\mathfrak{M}$. For example, we can use the following pair of definitions to strengthen the notion of measurable along the dimensions of (i) and (ii):

Definition 2. A cardinal $\kappa$ is $\lambda$-supercompact iff it is the critical point of a non-trivial elementary embeddings $j: V \longrightarrow \mathfrak{M}$, such that $j(\kappa)>\lambda$ and ${ }^{\lambda} \mathfrak{M} \subseteq \mathfrak{M}$ (i.e. $\mathfrak{M}$ is closed under $\lambda$-sequences).

Definition 3. A cardinal $\kappa$ is supercompact iff it is $\lambda$-supercompact for all $\lambda \in O n$.

The definition of supercompact uses the dimensions of (i) and (ii) to increase the strength of the embedding. We postulate a higher degree of similarity between $V$ and $\mathfrak{M}$ (in terms of closure under $\lambda$-sequences for the relevant $\lambda$ ), and stipulate that $j$ sends $\kappa$ above $\lambda$.

Standard discussions of middling large cardinals proceed from this template. However, we can generalise the construction to generic embeddings. Given a forcing construction adding a generic $G$ over a model $\mathfrak{N}$, a generic embedding is of the form $j: \mathfrak{N} \longrightarrow \mathfrak{M} \subseteq \mathfrak{N}[G]$. In other words, we begin to study embeddings from structures to inner models of their forcing extensions.

Recently, there has been an increased focus on such embeddings. Indeed, the study of generic embeddings has become widespread, as Foreman (in a Hanbook of Set Theory article on generic embeddings) illustrates ${ }^{26}$

"The main aim of the chapter is to illustrate that there is a coherent theory here, that there are unifying fundamental ideas that occur frequently in many different contexts. These include master condition ideals, natural and induced ideals, disjointing, self-genericity, the role of diagonal unions for representing Boolean sums, good elementary substructuresthe list is long." ([Foreman, 2010], p890)

Generic embeddings are thus useful for studying certain natural mathematical properties. Furthermore, the involvement of extensions in the consideration of embeddings provides an additional dimension in which we may vary the nature of the construction. Not only does the embedding depend upon the size of the domain and range of the embedding and where the ordinals are sent, but also on a third parameter:

(iii) The nature of the forcing required to define $j$

The fact that we have an extra dimension in which we can vary the structure of these kinds of embeddings makes them an intriguing subject matter. However, even more interesting is that the critical points of these axioms can be rather small. For example, we have:

Theorem 4. If $I$ is an $\omega_{2}$-saturated ideal on $\omega_{1}$ and $U$ is generic for the poset of $I$ positive sets, then in $V[U]$ the ultrapower $U l t(V, U)$ is well-founded and we get a $\operatorname{map} j: V \longrightarrow \mathfrak{M} \subseteq V[U]$ with $\operatorname{crit}(j)=\omega_{1}$ and $j\left(\omega_{1}\right)=\omega_{2}$.

\footnotetext{
${ }^{25}$ See [Kunen, 1971] for the result, and [Schindler, 2014] for a recent presentation.

${ }^{26}$ See also, [Foreman, 1986] for several key results, and [Foreman, 1998] for a more informal overview.

${ }^{27}$ For further exposition of this line of thinking, see [Foreman, 1998] and [Foreman, 2010].
} 
Such an embedding from $V$ to another model $\mathfrak{M}$ has $\omega_{1}$ as its critical point, far below the size of a measurable cardinal ${ }^{28}$ Despite the smallness of the critical points, however, these embeddings have a significant amount of large cardinal strength ${ }^{29}$ Thus, these embeddings provide significant combinatorial power whilst facilitating proof concerning small uncountable sets ${ }^{30}$

Moreover, it is not just through postulating the existence of embeddings directly between $V$ and models within forcing extensions that allow us to formulate new axioms with significant large cardinal strength. Through analysing properties of ordinals in extensions, we can come to characterisations of new varieties of cardinal.

An example of Schindler is especially pertinent here:

Definition 5. [Schindler, 2000] A cardinal $\kappa$ is remarkable iff in the $\operatorname{Col}(\omega,<\kappa)$ forcing extension $V[G]$, for every regular $\lambda>\kappa$ there is a cardinal $\lambda_{0}<\kappa, \lambda_{0}$ regular in $V$, and $j: H_{\lambda_{0}}^{V} \longrightarrow H_{\lambda}^{V}$ such that $\operatorname{crit}(j)=\gamma$ and $j(\gamma)=\kappa$.

We are able to characterise the notion of a cardinal being remarkable if, when we collapse all cardinals less than $\kappa$ to $\omega$ through forcing, in this $\operatorname{Col}(\omega,<\kappa)$ extension $V[G]$, for every regular $\lambda>\kappa$ in $V[G]$ there is a $V$-regular cardinal $\lambda_{0}<\kappa$ such that the heriditarily $\lambda_{0}$-sized sets elementarily embed into the hereditarily $\lambda$-sized sets. Thus, by studying how sets are embeddable in the extension, we are able to ascribe large cardinal properties to ordinals in $V$. The consistency strength of a remarkable cardinal for instance, while not strong enough to push us outside $V=L$, is substantially stronger than a weakly compact cardina ${ }^{31}$ The problem here is that we are predicating a large cardinal property of $\kappa$, an object in $V$, but using resources from extensions to define what it is to have said large cardinal property.

The case of class forcing is somewhat trickier, since it has received less attention than set forcing. However, some uses have been found in the literature; for example certain generic embeddings can be defined using class-sized stationary tower forcing (on the assumption that $V$ satisfies large cardinal properties) ${ }^{32}$

Moreover, one can show that class forcing facilitates the formalisation of different axioms that go beyond what can be captured by set forcing.

A good example here is:

\footnotetext{
${ }^{28}$ We know, for instance, that $\left|\omega_{1}\right| \leqslant|\mathcal{P}(\omega)|$, making it accessible. To get an idea of the scale of the difference, if $\kappa$ is measurable then it has to be an inaccessible limit of inaccessible cardinals.

${ }^{29}$ For example, the existence of both a saturated ideal on $\omega_{1}$ (and associated generic embedding) and a measurable cardinal implies the existence of an inner model with a Woodin cardinal, whereas the consistency strength of a measurable cardinal is far below that of a single Woodin. See [Steel, 1996] for details.

${ }^{30}$ Foreman, for example, writes concerning generic embeddings:
}

"The advantage of allowing the embeddings to be generic is that the critical points of the embeddings can be quite small, even as small as $\omega_{1}$. For this reason they have many consequences for accessible cardinals, settling many classical questions of set theory." ([Foreman, 2010], p887)

\footnotetext{
${ }^{31}$ Weakly compact cardinals are so named in virtue of being characterisable through compactness properties on infinitary languages. They admit of a diverse number of equivalent characterisations. For details, see [Kanamori, 2009].

${ }^{32}$ The following example is taken from [Larson, 2004], $\S 2.3$, p. 59. Suppose that $V$ contains a proper class of completely Jónsson cardinals. Letting $\mathbb{P}_{\infty}$ be the class tower forcing, and $G \subset \mathbb{P}_{\infty}$ be $V$-generic, and $V[G]$ be:

$$
V[G]=\bigcup_{\alpha \in O r d} L\left(V_{\alpha}, G \cap V_{\alpha}\right)
$$

there exists a generic embedding $j: V \longrightarrow V[G]$ such that for every $a \in \mathbb{P}_{\infty}, a \in G$ iff $j[\cup a] \in j(a)$. I thank [name removed for blind review] for bringing this example to my attention.
} 
Axiom 6. The Inner Model Hypothesis. Let $\phi$ be a parameter-free first-order sentence. If $\phi$ is true in an inner model of an outer model of $V$, then $\phi$ is already true in an inner model of $V$.

The Inner Model Hypothesis, as proposed by [Friedman, 2006], is meant to apply to arbitrary extensions of $V$ and raises difficult questions for how it might be coded ${ }^{33}$ However, note that it admits restricted forms (again, let $\phi$ be parameter free):

Axiom 7. The Set-Generic Inner Model Hypothesis. If $\phi$ is true in an inner model of a set-generic forcing extension $V[G]$ of $V$, then $\phi$ is already true in an inner model of $V$.

This admits of a natural generalisation:

Axiom 8. The Class-Generic Inner Model Hypothesis. If $\phi$ is true in an inner model of a class-generic forcing extension $V[G]$ of $V$, then $\phi$ is already true in an inner model of $V$.

Let us assume that a satisfactory coding mechanism for set forcings over $V$ is available. If the Set-Generic Inner Model Hypothesis is true (since we are interested in what is possible for a Universist, we will say little about its motivation here ${ }^{34}$ we get some consequences:

Fact 9. If the Set-Generic Inner Model Hypothesis is true, then $V \neq L=25$

However, though the Set-Generic Inner Model Hypothesis is sufficient to get us a certain richness of inner models (enough to break $V=L$ ) we get more if we allow class forcings. This is brought out in the following:

Fact 10. There is a model satisfying the Set-Generic Inner Model Hypothesis that does not satisfy the Class-Generic Inner Model Hypothesis ${ }^{36}$

It thus seems that class forcing is able to aid in the facilitation of formulating axioms that substantially go beyond those that are formalisable using set forcings. Again, since our current topic is to see ways in which the Universist might (rather than $m u s t$ ) lose insight, we will simply take it as given from this point on that class forcings provide a tool for proving theorems and formulating axioms concerning $V$.

\footnotetext{
${ }^{33}$ See Antos et al., S for discussion.

${ }^{34}$ One way of motivating these sorts of axioms is as absoluteness principles. Effectively, they say that $V$ already has witnesses for ways things 'could' be (in the sense of possibility in coded extensions).

Proof. Assume $V=L$ and that the Set-Generic Inner Model Hypothesis is true. Then there is an inner model of an outer model in which $V=L$ is false (the addition of a single Cohen real $x$ over $L$ to $L[x]$ will suffice, with the relevant inner model simply being the forcing extension $L[x]$ ). By the Set-Generic Inner Model Hypothesis there is an inner model of $L$ in which $V \neq L$. But $L$ is the smallest inner model, and so $V=L$ and $V \neq L, \perp$.

${ }^{36}$ I am grateful to [name removed for blind review] for discussion of the following:

Proof. Let $\mathfrak{M}$ be a model of $V=L$ containing a reflecting cardinal $\kappa$. (In the present context, $\kappa$ is a reflecting cardinal iff (i) $\kappa$ is regular, and (ii) whenever $\phi$ is a sentence with parameters from $V_{\kappa}$, if $\phi$ holds in some $V_{\alpha}$ (in $V$ ), then $\phi$ holds in some $V_{\beta}$ with $\beta<\kappa$. The definition is equivalent to saying that $\kappa$ is regular and $V_{\kappa}$ is $\Sigma_{2}$ elementary in $V$. In terms of consistency strength, it is stronger than inaccessible but weaker than Mahlo.) Next, perform the Lévy Collapse to move $\kappa$ to $\omega_{1}$. By the absorption properties attaching to the Lévy Collapse (see here [Cummings, 2010], §14) the extension $L[G]$ then satisfies the SetGeneric Inner Model Hypothesis. However, by |Jensen, 1972] one can define class-generic reals that are not set-generic. Let $H$ be a generic yielding such a real in an extension $L[H]$. We then note that $L[G]$ does not have an inner model with a real that is not set-generic over $L$, and so the Class-Generic Inner Model Hypothesis fails in $L[G]$.
} 


\subsection{The Naturalness Constraints}

Above, we saw how situating $V$ in a multiverse of universes obtained by forcing facilitates the proving of theorems and formulation of axioms beyond the merely number-theoretic. However, we also noted that the Universist could at least interpret this talk non-vacuously. How might we then combine the observation that forcing is more than a tool for proving independence into a genuine problem for the Universist?

The key notion will be the naturalness of interpretation provided. As we have just argued, the proving of theorems and formulation of axioms in the present context relates to more than the mere existence of models to required to prove relative consistency proofs. Forcing allows us to prove that certain uncountable cardinals are in fact equal (e.g. $\mathfrak{p}=\mathfrak{t}$ ), posit the existence of and study large cardinals (e.g. remarkable cardinals), and formulate axioms with consequences for the existence of real numbers and accessible cardinals (e.g. generic embeddings and variants of the inner model hypothesis).

We might then press the following idea: It is not enough to merely give some model or other in providing an interpretation of forcing. Since it seems that settheorists can use extensions of $V$ to reason about $V$, and that when performing this reasoning the relevant extensions appear to be bona-fide universes of set, we should want whatever interpretation we prescribe to be as faithful as possible to the set theorist's intuitive thinking. Perhaps under such constraints the Universist's interpretations will come out as deficient?

There are two immediate challenges to this line of attack:

(1.) The notion of what counts as a 'natural' or 'faithful' interpretation is imprecise.

(2.) It is unclear why the Universist cannot just reject this requirement on naturalness outright.

Both challenges can be answered, at least partially. Regarding (1.): While it is true to say that what the notion of being a 'natural', 'faithful', or 'semantically transparent' interpretation comes down to is going to be a difficult question to answer (in the sense of providing necessary and sufficient conditions) this does not mean that we have zero grip on the notion. In fact, the following all seem to be legitimate and precise constraints on ways in which an interpretation can be more or less natural:

(1.) (The Facetious Constraint.) The interpretation of ' $V$ ' could refer to $V, G$ to an actual generic outside $V$, and $V[G]$ to a literal extension of $V$.

(2.) The interpretation of ' $V$ ' in the construction could be $V$ itself.

(3.) More minimally, the interpretation of ' $V$ ' in the construction could satisfy the same first-order sentences as $V$.

(4.) One or both of the interpretations of ' $V$ ' and ' $V[G]$ ' could be well-founded, and hence admit of an absolute notion of being formed through transfinite iteration of a powerset-like operation.

(5.) The interpretations of ' $V$ ' and ' $V[G]$ ' could contain uncountable sets.

(6.) Each of ' $V$ ' and ' $V[G]$ ' could contain all the ordinals.

(7.) The structures denoted by each of ' $V$ ' and ' $V[G]$ ' could be two-valued. 
(8.) The movement between the interpretation of ' $V$ ' and ' $V[G]^{\prime}$ ' could be 'transparent', in the sense that whatever is denoted by ' $V[G]$ ' really is obtainable by the usual forcing idea of the addition of a generic to whatever is denoted by ' $V$ '.

(9.) Steps in proofs that use forcing constructions could be interpreted with the minimal amount of change, so additional or different steps do not need to be made to keep the proof in line with the interpretation.

We will refer to the project of trying to satisfy as many of the above desiderata as possible as satisfying the Naturalness Constraints. While we certainly do not contend that the list is complete, we think that the ease of providing such a preliminary list shows that we have some grip on the notion of what counts as more or less natural as an interpretation. The Facetious Constraint is so-named because it obviously cannot be satisfied by the Universist, but as we shall see shortly is helpful in clarifying the dialectic.

Regarding (2.): The question of whether or not the Universist should accept the Naturalness Constraints to any degree is going to be a difficult one. However, some points can be made in the favour of acceptance.

First, we might accept the Naturalness Constraints just for the sake of examining the opponents challenge on the strongest grounds possible. If the Universist can provide an interpretation that respects as many of the Naturalness Constraints as possible, this represents a more robust conclusion and so is philosophically desirable anyway.

Second, responding to the Naturalness Constraints is particularly dialectically effective given that some opponents to Universism seem to subscribe to similar ideas. Consider the following passages from Hamkins (an ardent anti-Universist):

"This abundance of set-theoretic possibilities poses a serious difficulty for the universe view, for if one holds that there is a single absolute background concept of set, then one must explain or explain away as imaginary all of the alternative universes that set theorists seem to have constructed. This seems a difficult task, for we have a robust experience in those worlds, and they appear fully set theoretic to us. The multiverse view, in contrast, explains this experience by embracing them as real, filling out the vision hinted at in our mathematical experience, that there is an abundance of set-theoretic worlds into which our mathematical tools have allowed us to glimpse." ([Hamkins, 2012], p. 418)

and

"...a set theorist with the universe view can insist on an absolute background universe $V$, regarding all forcing extensions and other models as curious complex simulations within it. (I have personally witnessed the necessary contortions for class forcing.) Such a perspective may be entirely self-consistent, and I am not arguing that the universe view is incoherent, but rather, my point is that if one regards all outer models of the universe as merely simulated inside it via complex formalisms, one may miss out on insights that could arise from the simpler philosophical attitude taking them as fully real." ([Hamkins, 2012], p. 426)

Part of Hamkins' point, it seems, is that while he acknowledges that the Universist can interpret forcing constructions, the interpretation provided is some how unnatural requiring "contortions" and not respecting the "mathematical experience". 
Better instead (he argues) to take a forcing multiversist position on which extensions are uncontroversially and easily available (indeed, the forcing multiversist immediately satisfies the Facetious Constraint). If the Universist can provide a response that satisfies as many of the Naturalness Constraints as possible, she would thus provide the strongest possible response to criticisms in the style of Hamkins.

Thirdly, the Naturalness Constraints chime well with things the Universist might be tempted to say anyway. A standard (but by no means the only) response one often receives when asking why one might hold the Universist picture is that it seems, given the iterative conception of set, that one is defining a unique structure. One simply iterates the powerset operation through the ordinals, thereby specifying $V$. Now, while the significance or effectiveness of such an argument is highly debatable, it nonetheless bears mentioning that the line of thinking relies on something like the Naturalness Constraints: It certainly seems as though we talk about a unique structure when we begin to study set theory through the iterative conception, and so the most natural interpretation (the Universist might argue) is that we do so. (Note that this remark (and the current debate) would apply equally well to theorists who do not hold that the universe has a definite height, but are nonetheless tempted by the idea that the powerset operation is definite.)

We thus believe that the Universist has at least some reason to try and provide an interpretation of forcing that respects the Naturalness Constraints. From now on we shall simply take it as an assumption that she would like to satisfy as much of the Naturalness Constraints as possible, whilst acknowledging that this is an area that merits further analysis.

As we shall see, extant interpretations of forcing constructions reveal that the Universist can satisfy a good deal of the Naturalness Constraints, although the various interpretations behave differently. First, however, let's examine a quick example to see how the Naturalness Constraints might exclude certain interpretations as fully satisfactory. Both remarkable cardinals and generic embeddings in fact admit of formulations internal to $V$, without mentioning forcing at all. Remarkable cardinals, for example, can be formulated in terms of embeddings between countable models and certain sets of particular hereditary cardinalities ${ }^{37}$ Generic embeddings can likewise be formulated by an internal to $V$ condition, usually in terms of the existence of particular ideals ${ }^{38}$ One might then just regard the formulation of remarkable cardinals and generic embeddings as a façon de parler for ascribing these internal-to- $V$ properties to certain objects.

Given the Naturalness Constraints, this strategy seems to perform relatively poorly. In particular, whilst there is no denying the mathematical interest of these equivalent

\footnotetext{
${ }^{37}$ See here [Schindler, 2001].

${ }^{38}$ Some remarks of Foreman are pertinent here:

"The language of ideals, together with the mechanics of forcing provide the same kind of vehicle for stating generalized large cardinal axioms in the language of set theory. Assuming the existence of a proper class of Woodin cardinals, Burke's Proposition...shows that every countably complete ideal is pre-precipitous. More directly: the existence of an elementary embedding $j: V \longrightarrow \mathfrak{M} \subseteq V[G]$ where $G \subseteq \mathbb{P}$ is generic and $j " \lambda \in \mathfrak{M}$ is easily seen to be equivalent to the existence of a $\mathbb{P}$-term for an ultrafilter $\dot{U} \subseteq \mathcal{P}(\mathcal{P}(\lambda))^{V}$ is normal for regressive functions in $V$ and fine and is such that there is no descending $\omega$-sequence of $U$-equivalence classes of functions from $V$. The idea of an induced ideal allows us to restate this combinatorially as a normal, fine, precipitous ideal $I$ on $\mathcal{P}(\lambda)$ such that the quotient algebra $\mathcal{P}(\mathcal{P}(\lambda)) / I$ inherits some of the properties of the original partial ordering $\mathbb{P}$. Finally, moving along the " $\mathrm{F}$ " axis [the nature of forcing required to define $j$ ] in the direction of greater strength, the saturation properties of ideals play exactly the same role for generalized large cardinals as ultrafilters do for conventional large cardinals." ([Foreman, 2010], p1128)
} 
characterisations, as an interpretation of the relevant forcing discourse there is no actual interpretation given to the forcing itself, no model similar to $V$ is extended, and there is no actual forcing occurring at all. In this way, the interpretation does not seem very natural; part of the insight gained from having these axioms is facilitating the study of objects of $V$ 'as if' they were situated in a forcing multiversist framework. Often the most natural context in which to define and study them (and the relevant associated objects internal to $V$ ) is the forcing perspective. An interpretation of forcing which can make use of this perspective thus gains more insight than one which cannot.

\section{Available interpretations}

Thus far we've argued that (1.) The Universist has reason to want to interpret forcing talk over $V$ (in order to study the objects of $V$ ) and (2.) Taking the Universist's opponent's complaints seriously depends on holding that some degree of 'naturalness in interpretation' is desirable. We will now analyse ways that we might code talk concerning forcing extensions, and see how they perform with respect to the Naturalness Constraints. We'll argue that different interpretations sacrifice different aspects of the Constraints, but that some are more serious than others. In the end we'll see that the so-called Boolean ultrapower map is especially natural for a certain class of forcings, and the countable transitive model strategy provides a method that satisfies many of the Naturalness Constraints across the board.

\subsection{The forcing relation}

We begin with a discussion of how we might try to capture claims concerning forcing extensions syntactically by defining a relation that captures the consequences of extensions without actually committing to the existence of any models. For forcing, this can be done by defining a so-called forcing relation. Roughly put, letting $\mathbb{P}=\langle P, \leqslant \mathbb{P}\rangle$ be a forcing poset, $p \in P$, and $\phi$ be in the forcing language for $\mathbb{P}{ }^{39}$, we can define a relation $p \Vdash_{\mathbb{P}}^{*}$ recursively ${ }^{40}$ One can then verify:

(1) If $\phi_{1}, \ldots, \phi_{n} \vdash \psi$ and $p \Vdash_{\mathbb{P}}^{*} \phi_{i}$ for each $i$, then $p \Vdash_{\mathbb{P}}^{*} \psi$.

(2) $p \Vdash_{\mathbb{P}}^{*} \phi$ for every axiom of ZFC.

(3) If $\phi\left(x_{1}, \ldots, x_{n}\right)$ is a formula known to be absolute for transitive models, then for every $p$ and all sets $a_{1}, . . a_{n} ; p \Vdash_{\mathbb{P}}^{*} \phi\left(\check{a}_{1}, \ldots, \check{a}_{n}\right)$ iff $1_{\mathbb{P}} \Vdash_{\mathbb{P}}^{*} \phi\left(\check{a}_{1}, \ldots, \check{a}_{n}\right)$ iff $\phi\left(a_{1}, \ldots, a_{n}\right)$ is true in $V$.

Essentially, $\Vdash_{\mathbb{P}}^{*}$ lets us talk about what would be satisfied in the extension $V[G]$ by analysing what sentences conditions $p \in P$ force. In particular, if we can show that there is a $p \in P$ such that $p \Vdash_{\mathbb{P}}^{*} \phi$, we can behave and talk as if such a forcing extension exists. By (3), any theorem proved 'in $V[G]$ ' will be verified by the check names and hence by specific sets in $V$. Similarly, if we wish to formulate an axiom about $V$ using a forcing extension, we can do so by finding a $p$ that forces the required sentence about objects in the ideal extension.

The use of the forcing relation is absolutely fine for relative consistency proofs. We know that if we can find a $p \in \mathbb{P}$ such that $p \Vdash_{\mathbb{P}}^{*} \phi$, then we cannot prove $\neg \phi$

\footnotetext{
${ }^{39}$ The forcing language of $\mathbb{P}$ is the collection of all formulas that can be formed by the usual logical operators from the language $\mathscr{L}_{\in}$ combined with a constant symbol for every name in $V^{\mathbb{P}}$ (the $\mathbb{P}$-names).

${ }^{40}$ See [Kunen, 2013] for details of the forcing relation and verification of the relevant proofs.
} 
(assuming the consistency of ZFC). There are, however, several problems with this approach to interpreting forcing talk.

First, there is a problem of scope. The difficulty lies in the fact that the forcing relation need not be definable when the forcing poset in question is proper-class-sized. For example, consider the following forcing:

Definition 11. Let $\mathfrak{M}$ be a model for ZFC. Then the Friedman poset (denoted by ' $\mathbb{F}^{\mathfrak{M} \prime}$ ') is a partial order of conditions $p=\left\langle d_{p}, e_{p}, f_{p}\right\rangle$ such that:

(i) $d_{p}$ is a finite subset of $\omega$.

(ii) $e_{p}$ is a binary acyclic relation relation on $d_{p}$.

(iii) $f_{p}$ is an injective function with $\operatorname{dom}\left(f_{p}\right) \in\left\{\varnothing, d_{p}\right\}$ and $\operatorname{ran}\left(f_{p}\right) \subseteq \mathfrak{M}$.

(iv) If $\operatorname{dom}\left(f_{p}\right)=d_{p}$ and $i, j \in d_{p}$, then $i e_{p} j$ iff $f_{p}(i) \in f_{p}(j)$.

(v) The ordering on $\mathbb{F}^{\mathfrak{M}}$ is given by:

$$
p \leqslant_{\mathbb{F} M} q \leftrightarrow d_{q} \subseteq d_{p} \wedge e_{p} \cap\left(d_{q} \times d_{q}\right)=e_{q} \wedge f_{q} \subseteq f_{p} .
$$

This defines a proper-class-sized partial order as the individual $f_{p}$ include every function from some finite subset of $\omega$ to a (sub)set of $\mathfrak{M}$, and hence there are properclass-many such ordered triples (relative to $\mathfrak{M}$ ). The partial order adds a bijection $F_{\mathbb{F}}$ between $\omega$ and $M$, and a relation $E_{\mathbb{F}} \in \mathfrak{M}[G]$ such that $\left\langle\omega, E_{\mathbb{F}}\right\rangle$ and $\langle M, \epsilon\rangle$ are isomorphic. If the forcing relation for $\mathbb{F}$ were definable, $\mathfrak{M}$ would then have access to its own truth definition (contradicting Tarski's Theorem) ${ }^{41}$

Thus we have:

Theorem 12. [Holy et al., 2016] (attributed to Friedman) $\Vdash_{\mathbb{F}}^{*}$ is not uniformly definable for $\mathbb{E}$.

Hence, there are forcings for which there is no definition of the forcing relation in the ground model. This is true, despite the fact that $\mathbb{F}^{\mathfrak{M}}$ itself is definable over $\mathfrak{M}$. If we wish to use $\mathbb{F}^{V}$ in proving facts about $V$ then, we cannot do so through consideration of a forcing relation in $V$. Since well-behaved forcings (so called 'tame' and 'pretame' class forcings) ${ }^{42}$ have definable forcing relations, we know that any extension using $\mathbb{F}$ must violate $\mathbf{Z F C}{ }^{43}$ One may feel that this provides a response: we should not consider such forcing arguments as legitimate for proving facts about $V$ because the resulting 'model' we are trying to talk about is pathological-it is non-ZFC-preserving.

We hold that the Universist should have little truck with such a claim. It is true that the resulting extensions are pathological in violating ZFC. However, we should note that (from the Universist's perspective) the whole enterprise with which we are engaged (namely considering extensions of $V$ ) is somewhat pathological: such sets cannot literally exist. We are rather trying to code in $V$ the effects of viewing $V$ as part of a forcing multiversist framework despite the fact that the relevant extensions do not exist (strictly speaking). Thus, there seems to be no objection to considering models where, say, there is a bijection between $\omega$ and $V$ (as is the case when forcing

\footnotetext{
${ }^{41}$ For the details of the proof, and further discussion of the Truth and Definability lemmas in context of class forcing, see [Holy et al., 2016].

${ }^{42}$ Pretameness implies the preservation of Replacement in a class forcing, and tameness additionally requires that the forcing preserve the Power Set Axiom. For details, see [Friedman, 2000].

${ }^{43}$ See [Holy et al., 2016].
} 
using $\mathbb{F}^{V}$ ). If consideration of such 'pathologies' has interesting consequences (such as the study of how truth predicates might behave) within $V$ then there seems little reason to prohibit their examination.

One might simply postulate that a definition can be given for $\mathbb{F}^{V}$ and other classsized partial orders with non-first-order definable forcing relations. Such definitions could not be first-order, but interestingly such a hypothesis fits naturally in the space of second-order set theories between NBG and MK. [Gitman et al., 2017] showed that the hypothesis that every class partial order has a forcing relation is equivalent to the principle that transfinite recursions of class relations for ordinal length are legitimate (so called 'ETR $R_{\text {Ord }}$ '). Whether this issue of scope can be assuaged thus depends precisely on one's attitude to second-order class theory. Presumably the Universist who wishes to try and interpret class forcing over $V$ has some sympathy with the use of second-order resources (since the classes added are often non-firstorder definable), and so we set the problem of scope aside.

Irregardless, the use of $\Perp_{\mathbb{P}}^{*}$ fares reasonably poorly with respect to the Naturalness Constraints. For many cases of forcing, reasoning syntactically about the forcing relation does not accord with the phenomenological character of the set theorist's reasoning. They wish to reason about sets which can be combinatorially manipulated, embeddings which move ordinals, and so forth. Thus, while ' $V$ ' is able to denote $V$ in this interpretation of forcing, there are no models involved (two-valued or otherwise), there is no actual forcing occurring, and no model which is actually extended. This issue is brought out clearly when we consider generic embeddings. These provide us with quintessentially combinatorial kinds of reasoning; we want to see what ordinals are moved by $j$ (and where) and what the structure of the remaining sets looks like given the existence of $j$. Here, however, the reasoning is fully syntactic; we analyse which formulas particular $p \in \mathbb{P}$ force, and so are explicitly not working with sets in the above manner. In this way, the use of the forcing relation, though both useful and of mathematical interest, leaves some philosophical issues untouched.

\subsection{Boolean-valued models}

Next, we consider the use of Boolean-valued models ${ }^{44}$ Starting with a forcing poset $\mathbb{P}$, we can find a separativ ${ }^{45}$ partial order $\mathbb{Q}$, equivalent to $\mathbb{P}$ for forcing, and a (unique up to isomorphism) Boolean completion of $\mathbb{Q}$ (denoted by ' $\left.\left.\mathbb{B}(\mathbb{Q})^{\prime}\right){ }^{46}{ }^{47}\right]$ We then consider the class of $\mathbb{B}(\mathbb{P})$-names (denoted by ' $\left.V^{\mathbb{B}(\mathbb{P})}\right)$, assign values from $\mathbb{B}(\mathbb{P})$ to atomic relations between them, and provide an inductive definition for the quantifiers 48

It is then routine to show that $V^{\mathbb{B}(\mathbb{P})}$ is a Boolean-valued model of ZFC. In particular every axiom (and hence every theorem) of $\mathbf{Z F C}$ has Boolean-value $1_{\mathbb{B}(\mathbb{P})}$ in $V^{\mathbb{B}(\mathbb{P})}$. Moreover, for the purposes of consistency proofs, we know that if we can assign $\phi$ a Boolean-value greater than $0_{\mathbb{B}(\mathbb{P})}$, then $\neg \phi$ is not a consequence of ZFC (as if $\neg \phi$ is a consequence of $\mathbf{Z F C}$, then $\phi$ receives Boolean value $0_{\mathbb{B}(\mathbb{P})}$ ). In fact, an

\footnotetext{
${ }^{44}$ The Boolean-valued approach was developed by Scott and Solovay, with additional contributions by Vopěnka (among others). See [Smullyan and Fitting, 1996], p. 273 for historical details and references.

${ }^{45}$ A partial order $\mathbb{P}=\left(P,<_{\mathbb{P}}\right)$ is separative iff for all $p, q \in P$, if $p \Varangle_{\mathbb{P}} q$ then there exists an $r \leqslant \mathbb{P} p$ that is incompatible with $q$.

${ }^{46}$ For details of Boolean algebras (from which our presentation is derived) see [Jech, 2002], Chapter 7. A discussion of Boolean completions is available in ibid. Chapter 14 .

${ }^{47}$ We will (mildly) abuse notation and use $\mathbb{B}(\mathbb{P})$ to refer to the relevant Boolean completion even when $\mathbb{P}$ is not separative (i.e. the Boolean completion obtained from a separative partial order $\mathbb{Q}$, such that $\mathbb{Q}$ is equivalent to $\mathbb{P}$ for forcing).

${ }^{48}$ See here, [Jech, 2002], Ch. 14.
} 
assignment of a Boolean value greater than $0_{\mathbb{B}(\mathbb{P})}$ to $\phi$ exactly mimics the satisfaction of $\phi$ in some $V[G]$, for $V$-generic $G$.

Thus, by discussing the Boolean-valued model $V^{\mathbb{B}(\mathbb{P})}$, we are able to capture the intra- $V$ content of talking about set forcing extensions of $V$. For example, suppose that we wish to show that the satisfaction of $\phi$ in a set forcing extension by $G \subseteq \mathbb{P} \in V$ has consequence $\psi$ within $V$. We can then take the Boolean completion $\mathbb{B}(\mathbb{P})$, and show that we can assign $\phi$ Boolean-value greater than $0_{\mathbb{B}(\mathbb{P})}$ in $V^{\mathbb{B}(\mathbb{P})}$. By tracing the Boolean-values back to $V$, we then know that $V$ satisfies $\psi$.

There are several problems with the use of Boolean-valued models, however. It has two particular limitations when it comes to forcing, despite its ability to capture satisfaction in forcing extensions.

The first is again a problem of scope. It is unclear how to interpret class forcing on the present approach. For, in class forcing, the relevant partial order $\mathbb{P}$ is properclass-sized, and hence unbounded in the $V_{\alpha}$. When defining the Boolean completion $\mathbb{B}(\mathbb{P})$ we then encounter a difficulty. The usual method for defining a Boolean completion is to find a separative partial order equivalent to $\mathbb{P}$ for forcing (known as the separative quotient), and embed it into a Boolean algebre ${ }^{49}$ Effectively, we add a bottom element and the required suprema to form $\mathbb{B}(\mathbb{P}){ }^{50}$

In the present context, however, it is unclear that this can always be done. Since the partial order always goes to the 'top' of $V$, one can not always assume that there will be space to add a bottom element and suprema. This is not to say that it is impossible to provide a Boolean completion for class forcings; as it turns out a class partial order has a class Boolean-completion in a model of MK precisely when all antichains are at most set-sized (known as the $\mathrm{Ord}$-chain condition) ${ }^{51}$ This does show, however, that the kinds of class forcings one can interpret using this technique are rather restricted.

However, putting aside the questions of scope here, we note that even for the forcings that can be interpreted this way, the Naturalness Constraints are left somewhat neglected. This is for similar reasons to the forcing relation. Though the approach is now somewhat model-theoretic, the two-valued aspect of the set theorist's reasoning is missed. When we reason with $V[G]$ it 'looks-like' we reason with a standard set-theoretic model, and the Boolean valued model $V^{\mathbb{B}}$ does not have this flavour-rather it looks like we reason with Boolean-valued 'probabilistic' sets (since, one can informally think of a Boolean-valued model $V^{\mathbb{B}}$ as assigning 'probabilities' from $\mathbb{B}$ to membership and equality). Moreover, again no model is actually extended in considering these constructions. To accord more fully with the Naturalness Constraints, we need a two-valued model that looks a lot like $V$, and also gets extended in the relevant construction.

\subsection{Boolean ultrapowers and quotient structures}

There are, however, ways of modifying Boolean-valued models to proper-class-sized two-valued structures. We provide an informal description of the technique to highlight philosophical issues, but it is developed in detail in [Hamkins and Seabold, 2012] (and referred to in Hamkins' paper on the multiverse [Hamkins, 2012]). Importantly, the method provides a way of finding models internal to $V$ that bear forcing relationships to one another. This is done by first taking an ultrafilter $U$ on the relevant

\footnotetext{
${ }^{49}$ More formally, for any set-sized partial order $\mathbb{P}$, there is a Boolean algebra $\mathbb{B}(\mathbb{P})$ and an embedding $e: \mathbb{P} \longrightarrow \mathbb{B}(\mathbb{P})^{+}$(where $\mathbb{B}(\mathbb{P})^{+}$is the set of non-zero elements of $\mathbb{B}(\mathbb{P})$ ) such that for $p, q \in \mathbb{P}:(\mathrm{i})$ if $p \leqslant \mathbb{P} q$, then $e(p) \leqslant \mathbb{B}(\mathbb{P}) e(q)$, (ii) $p$ and $q$ are compatible iff $e(p) \wedge e(q)$, and (iii) $\{e(p) \mid p \in \mathbb{P}\}$ is dense in $\mathbb{B}(\mathbb{P})$.

${ }^{50}$ For the full details, see [Jech, 2002], Chapter 14.

${ }^{51}$ See [Holy et al., 2017] for the result.
} 
Boolean-algebra $\mathbb{B}($ for convenience sake, we now drop the notation $\mathbb{B}(\mathbb{P})$ ) and constructing a particular ultrapower embedding $j_{U}$ (the so-called Boolean ultrapower map) between $V$ and an inner model $\check{V}_{U}$. When we then form the quotient structure $V^{\mathbb{B}} / U$ of $V^{\mathbb{B}}$ (formed by taking the standard quotient structure), we find an interesting relationship between $\check{V}_{U}$ and $V^{\mathbb{B}} / U: V^{\mathbb{B}} / U$ is precisely the forcing extension of $\check{V}_{U}$ by $U$. One can verify that $V^{\mathbb{B}} / U \models \mathbf{Z F C}$ and also that if $\phi$ has Boolean-value greater than $0_{\mathbb{B}}$ in $V^{\mathbb{B}}$, then $V^{\mathbb{B}} / U \models \phi$. In more formal terms, one can prove:

Theorem 13. [Hamkins and Seabold, 2012] The Naturalist Account of Forcing. If $V$ is the universe of set theory and $\mathbb{B}$ is a notion of forcing, then there is in $V$ a definable class model of the theory expressing what it means to be a forcing extension of $V$. Specifically, in the forcing language with $\in$, constant symbols $\check{x}$ for every $x \in V$, a predicate symbol $\check{V}$ to represent $V$ as a ground model, and a constant symbol $\stackrel{G}{G}$, the theory asserts:

(1) The full elementary diagram of $V$, relativised to the predicate $\check{V}$, using the constant symbols for elements of $V$.

(2) The assertion that $\check{V}$ is a transitive proper class in the (new) universe.

(3) The assertion that $\stackrel{G}{ }$ is a $\check{V}$-generic ultrafilter on $\check{\mathbb{B}}$.

(4) The assertion that the new universe is $\check{V}[G]$, and ZFC holds there.

Importantly, there is no need for the ultrafilter to be $V$-generic here, and hence $U$ can perfectly well be in $V$. In fact, when one constructs the Boolean ultrapower over some model of set theory $\mathfrak{M}=(M, E)$, the claim that $U$ is $\mathfrak{M}$-generic is equivalent to the Boolean ultrapower $j_{U}$ being trivial (i.e. letting $E_{U}$ be the 'membership' relation defined by the Boolean ultrapower, $j_{U}$ is an isomorphism between $\mathfrak{M}$ and $\left(\check{M}_{U}, E_{U}\right)$ ).

In the case where $j_{U}$ is non-trivial on $V$, we map $V$ to a subclass of itself (much as we do with a measurable cardinal embedding). Since $\breve{V}_{U}$ is not the whole of $V$ when $U$ is in $V$ (and hence not $V$-generic), it is possible for a set external to $\check{V}_{U}$ to be our generic for $\check{V}_{U}$. Our interpretation of forcing might then be as follows. We note that $V_{U}$, whilst not isomorphic to $V$, nonetheless looks a lot like $V$; it is a proper-classsized elementary extension of $V$. Instead of using the Boolean-valued model $V^{\mathbb{B}}$ (with its attendant difficulties regarding classicality and combinatorial properties), we could interpret our use of the term ' $V^{\prime}$ and ' $V[G]^{\prime}$ as $\check{V}_{U}$ and $V^{\mathbb{B}} / U$ respectively. We would then perform far better with respect to the earlier outline Naturalness Constraints, we have a two-valued structures, both proper-class sized, and there is actual model-theoretic extension of these structures occurring.

There are, however, several problems with this approach. We should first note that the use of Boolean ultrapowers and quotient structures suffers from the same problem of scope as the Boolean-valued model approach-since it depends on finding Boolean completions we will not be able to use the method for certain kinds of class forcing. We have, however, moved to an interpretation on which the reasoning is both two-valued and combinatorial, and on which a model very similar to $V$ is extended.

However, this construction can often come at the price of well-foundedness. Observe that if the Boolean ultrapower map is to be well-founded, it must elementarily embed $V$ into an inner model thereof, and hence must have a critical point $\kappa$ that is measurable. More precisely:

Theorem 14. [Hamkins and Seabold, 2012] If $U$ is an ultrafilter in $V$ on the complete Boolean algebra $\mathbb{B}$, then the following are equivalent: 
(1) $\check{V}_{U}$ is well-founded.

(2) $\check{V}_{U}$ is an $\omega$-model (i.e. has the standard natural numbers).

(3) $U$ meets all countable maximal antichains of $\mathbb{B}$ in $V$.

(4) $U$ is countably complete over $V$ (i.e. if $\left\langle a_{n}\right| n\langle\omega\rangle \in V$ is an $\omega$-sequence of $a_{n} \in U$, then $\left.\wedge_{n} a_{n} \in U\right)$.

(5) $U$ is weakly countably complete over $V$ (i.e. if $\left\langle a_{n} \mid n<\omega\right\rangle \in V$ is an $\omega$-sequence of $a_{n} \in U$, then $\left.\wedge_{n} a_{n} \neq \varnothing\right)$.

By (4), for the Boolean ultrapower to remain well-founded, we need significant large cardinal properties attaching to the completeness of the ultrafilter $U^{52}$ Recall that many of the generic embeddings we wished to talk about had very small critical points. We are now in a position to mention the following:

Fact 15. Let $U$ be an ultrafilter on a complete Boolean algebra $\mathbb{B} \in V$, and assume that $\check{V}_{U}$ is well-founded with $j_{U}$ such that $\operatorname{crit}\left(j_{U}\right)=\kappa$. Then $\check{V}_{U}$ cannot be used to interpret forcing constructions that change the structure of sets below $V_{\kappa}$ [3

The key philosophical consequence of this fact is that if we are to interpret a forcing construction that involves the structure of sets below a measurable cardinal, then the Boolean ultrapower cannot be well-founded. This creates serious problems with respect to the Naturalness Constraints; in virtue of our adherence to the iterative conception, one might think that well-foundedness is an especially important condition on structures conforming to our concept of set and qualifying as intended. But the above feature of the Boolean ultrapower violates this for many of our favourite forcing constructions. One cannot even add a single Cohen real without the Boolean ultrapower being non-well-founded. Moreover, we noted earlier that one of the key strengths of generic embeddings was to study 'small' accessible cardinals through the lens of large-cardinal-like embeddings. But, if we wish to move $\omega_{1}$ using a generic embedding, then any Boolean ultrapower construction interpreting the required forcing will have differences in relatively 'small' levels' (e.g. $V_{\omega_{1}+2}^{V_{U}}$ and $V_{\omega_{1}+2}^{V^{\mathrm{B}} / U}$ will have different sets as $\omega_{1}^{\breve{V}_{U}}$ is countable in $V^{\mathbb{B}} / U$ ). By the previous fact, there cannot be any difference in these levels without $\check{V}_{U}$ becoming non-well-founded; a severe philosophical limitation when we consider how a set theorist conceives of $V$ and $M$ as interellated well-founded structures in $V[G]$. Philosophically, this feature of forcing is especially important-it keeps the models well-founded and the resulting model also therefore conforms to the iterative conception of set; admitting an absolute notion of construction via transfinite iteration of a powerset-like operation ${ }^{54}$

\footnotetext{
${ }^{52}$ The existence of a countably complete non-principal ultrafilter is equivalent to the existence of a measurable cardinal.

Proof. It is a basic fact concerning measurable cardinals that if $\kappa$ is measurable and the critical point of some $j$, then $j$ preserves $V_{\kappa}$. (This is because if $j_{U}$ did not, there would be (per impossibile) a different critical point below $\kappa$-see for example [Schindler, 2014], p. 51.) In the case where $j_{U}$ is well-founded, since $\kappa$ is the critical point of $j_{U}$ we know that $j_{U}$ preserves $V_{\kappa}$. Thus one cannot add subsets below $V_{\kappa}$.

${ }^{54}$ Plausibly, this is a reason why the independence phenomenon has gained more traction in set theory than in arithmetic; the arithmetic sentences we know of that are independent are require very nonstandard models to witness the independence.
} 
This philosophical problem has a technical manifestation: While we can still trace results derived from the study of these embeddings back through $j_{U}$ to $V$, our reasoning in the embedded model will be severely limited. Since the non-well-foundedness of the models implies a high degree of non-absoluteness (the satisfaction predicate itself is not even absolute $e^{55}$, we cannot simply use many of our normal assumptions regarding the relationship of sets in $V$ to those in $\breve{V}_{U}$ and $V^{\mathbb{B}} / U$. For example, consider the following reasoning template (stated in naive forcing-language):

Example 16. Extend $V$ with a generic $G$ that adds subsets above the least measurable $\kappa$ to form $V[G]$. Now extend $V[G]$ with a generic $H$ adding subsets below $\kappa$ to form $V[G][H]$. Show that $V[G][H]$ satisfies some $\Delta_{1}$-formula $\phi$, and hence infer (by the absoluteness of $\Delta_{1}$ formulas for transtive models) that $V$ satisfies $\phi$.

How would this be interpreted using the Boolean ultrapower? Letting $\mathbb{B}$ be the Boolean algebra for $G$, and $U_{G}$ be the relevant ultrafilter, it is at least have possible that ' $V[G]^{\prime}$ can be interpreted as some well-founded inner model $V^{\mathbb{B}} / U_{G}$, extending $\breve{V}_{U_{G}}$. Letting $\mathbb{C}$ be the Boolean algebra corresponding to $H, j_{U_{G}}(\mathbb{C})$ be the image of $\mathbb{C}$ under $j_{U_{G}}$, and $U_{H}$ the corresponding ultrafilter on $j_{U_{G}}(\mathbb{C})$, we would then embed $V^{\mathbb{B}} / U_{G}$ into $\left(\overline{V^{\mathbb{B}} / U_{G}}\right)_{U_{H}}$, with forcing extension $\left(V^{\mathbb{B}} / U_{G}\right)^{j_{U_{G}}(\mathbb{C})} / U_{H}$. But now, while we would know that $\left(V^{\mathbb{B}} / U_{G}\right)^{j_{U_{G}}(\mathbb{C})} / U_{H} \models \phi$, it would also be a non-wellfounded structure, and so we cannot use the absoluteness and $\Delta_{1}$ nature of $\phi$ to infer $\phi$ holds of $\breve{V}_{U_{G}}$ (from where we could infer that $\phi$ holds of $V$ either by absoluteness or pulling back along $j_{U_{G}}$ ). Rather we have to slowly and carefully trace where each embedding takes us, what is satisfied where, and the nature of the models involved. Specifically, since $\left(V^{\mathbb{B}} / U_{G}\right)^{j_{G}}(\mathbb{C}) / U_{H} \models \phi$, we know by absoluteness there that $\left(\overline{V^{\mathbb{B}} / U_{G}}\right)_{U_{H}} \models \phi$ (since the two models are transitive relative to each other). Pulling back along $j_{U_{H}}$, we then have that $V^{\mathbb{B}} / U_{G} \models \phi$, and hence by absoluteness $\check{V}_{U_{G}} \models \phi$. (We can then pull back again or just use absoluteness to obtain $V \models \phi$.) The fact that the models are radically different in nature means that we have to be careful where we apply absoluteness, making sure that we pull back along the Boolean ultrapower before applying absoluteness to ensure no illegitimate uses of the absoluteness of $\Delta_{1}$-formulas. In the 'naive' reasoning, we just inferred from the fact that forcing keeps the models standard and $V[G][H] \models \phi$ that $V \models \phi$.

The philosophical point here is just the following; sometimes one might want to reason about $V$ as though it were in a multiverse framework, but the moves made sometimes depend upon the nature of forcing with respect to $V$. The fact that the Boolean ultrapower can fail to keep the models standard can result in many extrasteps needing to be made (rather than the single one required to interpret the forcing), and so represents a severe deformation of the set-theorist's intuitive thought.

It seems then, that though the use of Boolean ultrapowers and quotient structures provides a way of modifying Boolean-valued structures into a two-valued framework, the price can be high, presenting difficulties of both a technical and philosophical character. Interestingly, this opens a new area of enquiry; examine the cases where forcing and large cardinals combine to keep the ultrapower well-founded ${ }^{56}$ In these cases, the Boolean ultrapower fares excellently as an interpretation of forcing, suggesting that there are an especially 'natural' class of forcing constructions for the Universist. However, as a general technique for interpreting forcing constructions

\footnotetext{
${ }^{55}$ For details, see [Hamkins and Yang, 2013]. To give an example of just how extreme the phenomenon is, one can have two models that have the same objects as natural numbers, but disagree about whether a particular (non-standard) $n$ is odd or even.

${ }^{56}$ See [Hamkins and Seabold, 2012] for discussion.
} 
within a Universist framework, the technique falls short ${ }^{57}$

\subsection{Countable transitive models}

Examining the Naturalness Constraints, we can see that given that (i) we actually want a model to be extended in a forcing-like way, (ii) these models should be twovalued, and (iii) we would like the relevant models to satisfy as much as possible of the theory of $V$, the strategy of interpreting forcing constructions via the use of a Boolean ultrapower got a lot right. It suffered two problems, however; one of scope (interpreting class-sized Boolean algebras) and one of how the ultrapower behaved with respect to 'small' sets. This is what we'll try to remedy by analysing how we might use countable transitive models in interpreting forcing. As we'll see, there are several respects in which the countable transitive model strategy performs better with respect to the Naturalness Constraints, but nonetheless this comes at a price (specifically concerning uncountable sets). We'll conclude that the countable transitive model strategy represents an (if not the most) attractive way of interpreting forcing using the symbol ' $V$ ', however for certain applications the Boolean-ultrapower strategy is especially natural.

The countable transitive model strategy comes in several forms. Initially, the method was designed to deal with the apparently problematic metamathematics of forcing from within ZFC. Given a desired relative consistency proof of some sentence $\phi$, we assume that $\phi$ (respectively $\neg \phi$ ) is provable from ZFC. Since proofs are finite, we then know (by the Reflection, Löwenheim-Skolem, and Mostowski Collapse theorems) that there is a countable transitive model satisfying $\phi$, and furthermore this countable transitive model can be arranged so as to have enough structure to enable forcing. Since the model is countable, we know that generics are available $\sqrt{58}$ and can force to obtain $\neg \phi$ (whilst preserving the relevant fragment of ZFC), contradicting the claim that (the relevant fragment of) ZFC proves $\phi 59$

\footnotetext{
${ }^{57} \mathrm{~A}$ side remark should be made here about previous philosophical discussion concerning the Boolean ultrapower. Hamkins takes the Boolean ultrapower to show that the Universist can simulate forcing within her framework, and so should be moved to accept the existence of forcing extensions intuitively understood, and hence the falsity of her position. Koellner provides a response along the lines some of our observations here:

"There are three important things to note about $\left[V^{\mathbb{B}} / U\right]$-it need not be transitive, it need not be well-founded, it is a definable class in $V$. For all three reasons it is as non-standard a model of set theory...one sees by construction that the model produced is not of the appropriate type to count as the universe of sets." ([Koellner, 2013], pp. 19-20)

Our point is just the following: Even if the Universist should (plainly) assert that neither $\check{V}_{U}$ nor $V^{\mathbb{B}} / U$ is the universe of sets, it can sometimes provide a very close simulation of forcing claims. Hamkins takes this to show that the Universist is wrong. We push this in the other direction: It shows that the Universist can sometimes capture much of the insight that is meant to be an advantage of the forcing multiversist's position.

${ }^{58}$ Assuming, of course, that $V$ is uncountable.

${ }^{59}$ Formally, there are two main ways of executing the strategy (see |Kunen, 2013], IV.5.1 for details):

Suppose that we wish to prove that some statement $\phi$ is independent from ZFC. We then suppose that $\phi($ or $\neg \phi)$ has a proof in ZFC (from now on we only consider the case where we wish to show that $\neg \phi$ is unprovable). If $\neg \phi$ were provable, we would have $\mathbf{Z F C}+\phi \vdash 0=1$. Since proofs are finite, we then know that this proof would only use a finite set of axioms of ZFC (let it be denoted by ' $\Gamma$ '). We then know (by the forcing method) that given such a $\Gamma$, there is a larger finite set of axioms of $\mathbf{Z F C}$ (let it be denoted by ' $\Lambda$ ') such that ZFC proves the conditional:

"If there is countable transitive model for $\Lambda$, then there is a countable transitive model for $\Gamma+\phi . "$

However, now we can use the Reflection Theorem, Löwenheim-Skolem Theorem, and Mostowski Collapse Lemma to then obtain a countable transitive model $\mathfrak{N}$ for $\Lambda$, and hence have a countable transitive model for $\Gamma+\phi$, contradicting our supposition that $\Gamma+\phi \vdash 0=1$.
} 
Such methods are fine as far as they go. For the purpose of allowing us to analyse forcing arguments establishing relative consistency proofs from within ZFC the technique performs adequately; any inconsistency of $\phi$ with ZFC could be traced to a countable model, and the relevant forcing argument shows that there is no such inconsistency (on the assumption that ZFC itself is consistent).

However, while $V$ presumably satisfies ZFC and its finite fragmets for the Universist, there is no guarantee that a given countable transitive model of (a fragment of) ZFC represents $V$ with respect to first-order truth in general. While we can, for any appropriate given finite set $\Gamma$ of sentences satisfied by $V$, assume that there is a countable transitive model $\mathfrak{M}$ satisfying $\Gamma, V$ can (and indeed will) satisfy sentences beyond those in $\Gamma$, and there may be sentences such that $V \models \phi$ and $\mathfrak{M} \models \neg \phi$. In order then to mimic the behaviour of $V$ as closely as possible (and in line with the Naturalness Constraints) we would like a countable transitive model $\mathfrak{M}$ that resembles $V$ sufficiently well to allow us to interpret forcing over $V$ as closely as possible.

This can be achieved by assuming that there is a countable transitive model elementarily equivalent to $V$ for first-order truth (we will denote such a model with the letter ' $\left.\mathfrak{V}{ }^{\prime}\right){ }^{60}$ We can then interpret any forcing over this model, formulating axioms and proving theorems concerning $\mathfrak{V}$ (over which generics abound) and then, by the elementary equivalence, exporting the relevant consequences back to $V$ proper.

For example, if we wish to formulate an axiom that uses an extension (say a generic embedding), we simply formulate it as concerned with $\mathfrak{V}$ (where extensions are uncontroversially available) and then know that any first-order consequence of the axiom true in $\mathfrak{V}$ is also true of $V$. If an ideal of the required kind $I$ exists in $V$, then we will have a corresponding object $I^{\prime} \in \mathfrak{V}$, and then there will be the required embedding $i^{\prime}: \mathfrak{V} \longrightarrow \mathfrak{M} \subseteq \mathfrak{V}[G]$.

This strategy seems to resolve some of the problems of our previously considered accounts. First, regarding class forcing, we remarked earlier that we would like to interpret class forcings that lack a definable forcing relation, do not necessarily satisfy ZFC, or add classes but not sets. All these can be easily interpreted for $\mathfrak{V}$ : Letting $\mathfrak{V}=(\mathbf{V}, \in)$, we can consider models of the form $\mathfrak{V}^{\prime}=\left(\mathbf{V}, \in, \mathcal{C}^{\mathfrak{V}}\right)$, where $\mathcal{C}^{\mathfrak{V}}$ is some countable collection of classes for $\mathfrak{V}$. We can then perfectly well consider extensions that add classes to $\mathcal{C}^{\mathfrak{V}}$ without adding sets to $\mathfrak{V}$, and /or ones in which the the forcing relation is not definable over $\mathfrak{V}$, and first-order consequences ${ }^{61}$ thereby derived in $\mathfrak{V}$ can be transferred back to $V$. Similarly, since $\mathfrak{V}$ is very small by the standards of $V$, there is no obstacle to defining meaningful notions of Boolean completions for class

\footnotetext{
Alternatively, we could expand $\mathscr{L}_{\in}$ to $\mathscr{L}_{\in, C, F}$ by two constant symbols $C$ and $F$. We then add axioms to ZFC as follows (for this specific approach, see [Shoenfield, 1967] as well as [Kunen, 2013]):

Definition 17. ZFC* is a system in $\mathscr{L}_{\in, C, F}$ with the following axioms:

1. ZFC

2. $C$ is a transitive set.

3. $F$ is a bijection from $\omega$ onto $C$.

4. $\phi^{C}$ for every axiom $\phi$ of ZFC (note that, by Gödel's Second Incompleteness Theorem, this is an axiom scheme)

We know (by the Reflection Theorem, Löwenheim-Skolem Theorem, and Mostowski Collapse Lemma)

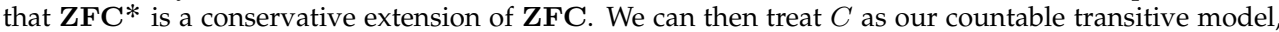
and conduct our construction there.

${ }^{60}$ Uses of this idea for developing axioms have been explored by Friedman and collaborators in [Arrigoni and Friedman, 2013], [Antos et al., 2015], and [Friedman, F].

${ }^{61}$ In this paper we restrict only to first-order consequences. Salient here is that the use of countable transitive models can also be applied to higher-order axioms that use extensions (such as those mentioned in [Friedman, F]).
} 
forcings over $\mathfrak{V}$ (though they might not be in $\mathfrak{V}$ ), since we can use the resources in $V$ external to $\mathfrak{V}$. Moreover, since generics are freely available over $\mathfrak{V}$ one does not need the Boolean algebras to interpret the forcing (though their study might nonetheless carry mathematical interest).

It seems then that the countable transitive model strategy is at least flexible concerning interpretation of forcing, providing a uniform way of interpreting the constructions. But how does it fare with respect to the Naturalness Constraints? Here, it also has a number of pleasing philosophical features. $\mathfrak{V}$ in many ways very closely resembles $V$; it is a two-valued structure that satisfies the same first-order sentences as $V$ and (in contrast with certain applications of the Boolean ultrapower) is really a well-founded structure (and so forms a genuine cumulative hierarchy). In this way, $\mathfrak{V}$ provides a miniature 'picture' of $V$ inside $V$, one which can be freely reasoned about using extensions. Moreover, once we have interpreted ' $V$ ' as ' $\mathfrak{V}$ ' in a forcing construction, interpretation is completely transparent; $\mathfrak{V}$ really is extended by some $G$ to form $\mathfrak{V}[G]$, and $\mathfrak{V}[G]$ has all the properties we would expect it to have: It is a well-founded cumulative hierarchy obtained from $\mathfrak{V}$ by forcing with $G$.

There are some drawbacks concerning the Naturalness Constraints, however. Unlike the Boolean ultrapower, $\mathfrak{V}$ cannot contain all the ordinals or uncountable sets. We think that preservation of well-foundedness is preferable to containing uncountable sets. This is in part for philosophical reasons; a well-founded model is one that is obtained by admitting a genuine powerset-like operation transfinitely, and thus comes close to the iterative conception in an absolute sense. A non-wellfounded model, even one containing uncountable sets, only has $V_{\alpha}$ internally (since the model will still satisfy the theorem of ZFC that every set belongs to some $V_{\alpha}$ ), but actually admits of a descending membership sequence and so cannot be said to come close to satisfying the iterative conception in any absolute sense. To our mind, having an absolute notion of the iterative conception should be privileged over the existence of actual uncountable sets-the former concerns the fundamentals of our contemporary conception of set whereas the latter is a result of our conception of set (as evidence for this, consider the fact that we call the Axiom of Foundation an axiom, whereas Cantor's Theorem is called a theorem) ${ }^{62}$ We might thus feel that it is more important for the former to be satisfied in an absolute manner than the latter (it is more important that our model actually be a well-founded cumulative hierarchy than actually contain uncountable sets). Moreover, a well-founded model (even a countable one) can have (a subclass of) real membership as its membership relation, whereas a non-well-founded model cannot. Other reasons are more technical; as we noted earlier (when considering repeated applications of forcing to form $V[G][H]$, absoluteness inferences concerning $\Delta_{1}$-formulas, and the Boolean ultrapower) one of the salient features of forcing is that it keeps the relevant model standard relative to the ground model. In the context of the use of countable transitive models then, repeated applications of forcing will always keep the model well-behaved with respect to the iterative conception (and subsequent uses of absoluteness), whereas this is not the case when the possibility of non-well-foundedness is introduced (as we saw with the abstract example of $V[G][H]$ earlier).

We thus arrive at the following picture; the Boolean ultrapower is especially natural when one can keep it well-founded. However, the countable transitive model strategy performs reasonably well (given the iterative conception) across the board. We suggest then that whilst the Boolean-ultrapower provides interesting questions for exploring Universism and forcing, if a uniform interpretation of forcing is de-

${ }^{62}$ [Koellner, 2014], §1.4.1 makes a similar point concerning a different theorem (The Hydra Theorem) and axiom (that $\epsilon_{0}$ is well-ordered). 
sired, the countable transitive model strategy fares better.

\section{Countable transitive models and Universism}

Thus far, we have argued that a modification of the countable transitive model strategy is reasonably philosophically virtuous, at least as far as the Naturalness Constraints are concerned. We are not done yet, however. Critics of Universism (such as [Hamkins, 2012]) are well aware of the possibility of both a countable transitive model of ZFC, and well as one elementarily equivalent to $V{ }^{63}$ Hamkins has two main objections against the use of countable transitive models (especially with regards to forcing constructions).

First, he complains that the countable transitive model strategy is not broad enough, since it only accounts for forcing over certain models of set theory, whereas other methods (such as the Boolean ultrapower) can interpret (set) forcing over any model ${ }^{64}$

Hamkins' second complaint surrounds the metamathematical commitments of the theorist endorsing the countable transitive model strategy. In order to interpret forcing over a countable transitive model, Hamkins argues, we have to assume the existence of a countable transitive model of ZFC. But this takes us somewhat beyond the consistency strength of our canonical set theory (i.e. ZFC); in fact the existence of a countable transitive model of ZFC implies the consistency of theories obtained by transfinite iterations of the consistency operator (so $C o n(\mathbf{Z F C}$ ), $\operatorname{Con}(\operatorname{Con}(\mathbf{Z F C}))$, and so on transfinitely). In this way, he argues, the Universist is forced to pay a metamathematical tax in implementing the countable transitive model strategy 65

We find each of Hamkins' objections unconvincing from the Universist's perspective. Concerning the scope of the countable transitive model strategy: Whilst Hamkins is, as a matter of mathematical fact, correct that if we interpret widthextending constructions as concerned with countable transitive models, our interpretation of extensions will not apply to arbitrary models, in the present context this does not motivate the claim that insight might be missed. In particular, if we slowly work through the dialectic, we see that the main difficulties we noted were (i) to provide an interpretation of of axioms mentioning extensions, (ii) to explain why

\footnotetext{
${ }^{63}$ For the former claim see [Hamkins, 2012], and for the latter claim [Hamkins, 2003].

${ }^{64}$ See for example, the following remarks:

"There are a number of drawbacks, however, to the countable transitive ground model approach to forcing. The first drawback is that it provides an understanding of forcing over only some models of set theory, whereas other accounts of forcing allow one to make sense of forcing over any model of set theory. With the countable transitive model approach to forcing, for example, the question "Is $\phi$ forceable?" appears sensible only when asked in connection with a countable transitive model $M$, and this is an impoverishment of the method." ([Hamkins, 2012], p421)

${ }^{65}$ For example, Hamkins argues as follows:

"A second drawback concerns metamathematical issues surrounding the existence of countable transitive models of $\mathbf{Z F C}$ : the basic problem is that we cannot prove that there are any such models, because by Gödel's Incompleteness Theorem, if ZFC is consistent then it cannot prove that there are any models of $\mathbf{Z F C}$ at all. Even if we were to assume Con (ZFC), then we still can't prove that there is a transitive model of ZFC, since the existence of such a model implies $\operatorname{Con}(\mathbf{Z F C}+\operatorname{Con}(\mathbf{Z F C}))$, and the consistency of this, and so on transfinitely...As a result, this approach to forcing seems to require one to pay a sort of tax just to implement the forcing method, starting with a stronger hypothesis than one ends up with just in order to carry out the argument." ([Hamkins, 2012], p421)
} 
theorems making use of extensions were acceptable for proving facts about $V$, and do so (iii) in a way that accounts for the majority of our intuitive thinking concerning extensions. For these purposes, the countable transitive model strategy is reasonably satisfactory as it stands. It is uncontroversial for a Universist that some models cannot literally be extended in width, indeed it is part of their view that width extension is impossible for some models (such as $V$ or one of its initial segments). Whilst it is true that certain constructions (such as use of the forcing relation or Boolean ultrapower map) permit intrepretation of forcing extensions where ' $V$ ' denotes $V$, some uncountable class, or possibly even a non-well-founded structure, we deny that this represents an impoverishment of the countable transitive model strategy. Those techniques are always available in cases where the countable transitive model does not apply (such as in the case of a non-well-founded proper class structure). We do not wish to argue that the countable transitive model strategy is superior in every case, rather we wish to analyse what is possible given the Naturalness Constraints and argue that the countable transitive model strategy is better for interpreting a certain class of extensions (namely when we wish to talk about $V$ using well-founded and transitive extensions). Our point is just that if we are concerned with $V$, the countable transitive model strategy provides a way of interpreting this practice in line with the Naturalness Constraints. Thus, unless the Universist is particularly wedded to the existence of uncountable sets in her interpretation of forcing, Hamkins' complaint fails to gain dialectic traction.

These points do nothing to assuage Hamkins' second worry, however. There is still the charge that the Universist pays a problematic metamathematical tax. Of course, one blunt response would be to say that the tax is worth it, so it should be paid. We wish to see if we can do better, however.

There are at least two ways in which we might take the Universist to have to pay a tax she would rather not. The first is a formal one: it might be the case that she must use assumptions of greater strength than ZFC in executing the strategy. Second, she might be paying an ontological tax, in that she has to commit herself to the existence of objects to which she would rather not.

Regarding the formal tax, Hamkins is correct that baldly asserting the existence of a countable transitive model of ZFC (of which presumably $\mathfrak{V}$ is one) implies $\operatorname{Con}(\mathbf{Z F C})$, $\operatorname{Con}(\operatorname{Con}(\mathbf{Z F C}))$, and so on, transfinitely. A response here is quick, however. The extent to which we move beyond $\mathbf{Z F C}$ is very dependent upon how we formulate the axiom. If we write:

Axiom 18. There exists a countable transitive model $\mathfrak{V}$ such that $\mathfrak{V} \models \mathbf{Z F C}$.

we would indeed go beyond ZFC. Indeed, adding a constraint that $\mathfrak{V}$ satisfies all the same sentences as $V$ would yield a contradiction by Tarski's Theorem on the undefinability of truth. We could instead formulate the axiom that there is a $V_{\alpha}$ which is $\Sigma_{\omega}$-elementary in $V$; a very slight extension of the provable Lévy Reflection Principle, and then Skolemise over $V_{\alpha}$. Much like the assumption of a transitive model of ZFC this assumption is very weak (well below an inaccessible). However, we can do even better formally. We can formulate the claim that there is a countable transtive model elementarily equivalent to $V$ via the use of the following axiom scheme (as noticed by [Feferman, 1969|):

Definition 19. Let $\mathscr{L}_{\epsilon, \overline{\mathfrak{V}}}$ be the language $\mathscr{L}_{\epsilon}$ augmented with a single constant symbol $\overline{\mathfrak{V}} . \mathbf{Z F C}{ }^{\overline{\mathfrak{V}}}$ is then a theory in $\mathscr{L}_{\in, \overline{\mathfrak{V}}}$ with the following axioms:

(i) ZFC 
(ii) $\overline{\mathfrak{V}}$ is countable and transitive.

(iii) For every $\phi$ in $\mathscr{L}_{\epsilon}, \phi \leftrightarrow \phi^{\overline{\mathfrak{V}}}$ (by Tarski's Theorem, this is an axiom scheme).

By replicating the proof of the Reflection Theorem for $\mathbf{Z F C}^{\overline{\mathfrak{V}}}$, we know that $\mathbf{Z F C}^{\mathfrak{V}}$ is a conservative extension of $\mathbf{Z F C}{ }^{66}\left[{ }^{67}\right.$ However, $\mathfrak{V}$ then satisfies exactly the same parameter-free first-order sentences of $\mathbf{Z F C}$ as $V$. When conducting a forcing proof in ZFC (or some extension thereof), we can use the usual trick of reflecting a finite set of axioms, but without loss of generality assume that they are reflected to $\mathfrak{V}$ to ensure enough similarity to $V$ and conformity with the Naturalness Constraints. Whilst we can only see from the metatheory (without a non-conservative extension of ZFC) that $\mathfrak{V}$ is a countable transitive model elementarily equivalent the $V$, this is to be expected: $V$ cannot have access to its own truth definition. Moreover, the Universist is no stranger to accepting the use of metatheoretical talk in her set-theoretic reasoning (for example: “ $V \models \mathbf{Z F C}$ ").

So it seems that we need not pay a significant formal price when interpreting width extensions with countable transitive models. It remains to be seen that the Universist need not pay an ontological price. To what extent does the existence of a countable transitive model elementarily equivalent to $V$ represent a substantial ontological presupposition for the Universist? We shall argue that the Universist already has good reasons to accept the existence of such a model given her commitment to the existence of a unqiue and maximal proper class model of set theory. We shall pursue two strategies here: (1) examining Skolem functions in the Universist's metatheory, and (2) the use of a truth predicate.

For (1) we start by examining an informal argument of Coher ${ }^{68}$ for the existence of a countable transitive model of $\mathbf{Z F}$ :

"The Löwenheim-Skolem theorem allows us to pass to countable submodels of a given model. Now, the "universe" does not form a set and so we cannot, in $\mathbf{Z F}$, prove the existence of a countable sub-model. However, informally we can repeat the proof of the theorem. We recall that the proof merely consisted of choosing successively sets which satisfied certain properties, if such a set existed. In $\mathbf{Z F}$ we can do this process finitely often. There is no reason to believe that in the real world this process cannot be done countably many times and thus yield a countable standard model for ZF." ([Cohen, 1966], p79)

While Cohen is primarily interested in the existence of a countable transitive model for ZF, we can import his argument to the case of $V$ as follows. When considering finite subsets $\Gamma$ of sentences in $\mathscr{L}_{E}$ satisfied by $V$ we move to a countable transitive model of $\Gamma$ by using the Reflection Theorem to find a $V_{\alpha} \models \Gamma$. We then use AC

\footnotetext{
${ }^{66}$ Proof Sketch. Begin by replicating the usual proof of the Reflection Theorem for $\mathbf{Z F C} \overline{\mathfrak{V}}$. Suppose then that $\phi \in \mathscr{L}_{\in}$ and $\mathbf{Z F C} \mathbf{V}^{\overline{\mathfrak{V}}} \vdash \phi$. Then there is a finite set of axioms $\Gamma$ of $\mathbf{Z F C}{ }^{\overline{\mathfrak{V}}}$, such that $\Gamma \vdash \phi$. Let $\psi$ be the conjunction of all axioms of $\mathbf{Z F C}{ }^{\overline{\mathfrak{V}}}$ in $\Gamma$ containing $\overline{\mathfrak{V}}$, and $\chi$ be conjunction of all axioms of $\mathbf{Z F C}$ in $\Gamma$ not containing $\overline{\mathfrak{V}}$. Without loss of generality, we may then assume that $\Gamma=\{\psi, \chi\}$. $\psi$ is then a sentence which states that there is a countable transitive model of some finite list of $\mathbf{Z F C}$ axioms $\Lambda$, and $\chi$ is a finite part of $\mathbf{Z F C}$. Since $\mathbf{Z F C}$ already proves that there is a countable transtive model for $\Lambda$ (by the Reflection Theorem and Mostowski Collapse Lemma in ZFC), and hence proves $\chi \wedge(\exists \mathfrak{M}) \mathfrak{M} \models \Lambda$, we have $\mathbf{Z F C} \vdash \phi$. Many thanks to [names removed for blind review] for pointing out to me this theorem of Feferman, and for discussion of the proof.

${ }^{67}$ It is interesting to note that [Hamkins, 2003] discusses this formulation in depth, and is well aware of the conservativity result. It is perhaps the metatheoretical character of the claim that $\mathfrak{V}$ satisfies $\mathbf{Z F C}$ that explains the absence of this strategy when considering countable transitive models in [Hamkins, 2012].

${ }^{68}$ Similar arguments (from a non-Universist perspective) are available in Arrigoni and Friedman, 2013.
} 
to find a set of functions $F^{V_{\alpha}}$ such that for every existential statement $\exists x \phi(x)$ true in $V_{\alpha}$, there is an $f_{\phi} \in F$ that picks a single witness $a$ such that $\phi(a)$ holds. We then form a countable $\mathfrak{M} \models \Gamma$. Since the relation (i.e. $\in$ ) on $V_{\alpha}$ is extensional, well-founded, and set-like, so is the relation on $\mathfrak{M}$, and we then use the Mostowski Collapse Lemma to collapse to obtain a countable transitive model $\mathfrak{M}^{\prime} \models \Gamma$. Turning now to $V$, we simply note that $V$ is one model of ZFC among many. Thus, we can posit the existence of a set of Skolem functions $F^{V}$ for $V$ (by 'choosing ${ }^{69}$ a witness for the countably many existential statements satisfied by $V$ with a countable sequence of choices). Then, by Skolemising and Collapsing, we obtain a countable transitive model $\mathfrak{V}$ that satisfies exactly the same parameter-free first-order sentences as $V$. The main issue here is that, by Tarski's Theorem on the undefinability of truth, that while $V$ can see $F^{V}$, it does not know that $F^{V}$ provides its own set of Skolem-functions. Thus, by adopting this informal and metatheoretic version of the Lowenheim-Skolem Theorem, we justify the existence of a countable transitive model for $V$. Again, we note that the Universist should not be bothered by making these various metatheoretic claims in virtue of the things she wants to say already concerning her own view.

Though the Cohen-style argument depends on only mild metatheoretic assumptions (the possibility of transferring a countable sequence of choices in the metalanguage to the existence of Skolem functions in $V$ ), it nonetheless depends upon a certain amount of combinatorial reasoning outside the object language. We might, therefore, find a more logical approach desirable. Here we find a role for the use of a truth predicate. We begin by noting that the Universist already makes claims concerning first-order parameter-free truth in $V$. For example:

Example 20. "In $V$ every sentence is either true or false."

Example 21. "Every axiom of ZFC is true in $V$. "

Given that she would like some formal apparatus for talking about her own view, it is natural for her to countenance the existence of a truth predicate for talking about her own view (otherwise she ends up baldly stating the sentences in question, rather than the truth thereof). We therefore make the following 70

Definition 22. Let $\mathbf{Z F C}_{T r}$ be the result of adding to $\mathbf{Z F C}$ a truth-predicate $T r$ for first-order parameter-free truth, such that $\operatorname{Tr}\left({ }^{\circ} \phi^{\top}\right)$ holds iff $V \models \phi$, adding the Tarskibiconditionals, and permitting the use of $\operatorname{Tr}$ in the Comprehension and Replacement schema.

Such a theory represents a very minimal formalisation of the first-level of typed truth over $V$ for the Universist. We now mention the following:

Fact 23. $\mathbf{Z F C} \mathbf{C}_{T r}$ proves that there is a countable transitive model elementarily equivalent to $V$ for first-order parameter-free truth in $\mathbf{Z F C}{ }^{71}$

Thus, if we permit the addition of a truth predicate for $V$ to $\mathbf{Z F C}$, the use of which a Universist may well accept (given the other presuppositions of her view),

\footnotetext{
${ }^{69}$ As Cohen notes, this is not possible in $\mathbf{Z F}(\mathbf{C})$ by Gödel's Second Incompleteness Theorem.

${ }^{70} \mathrm{I}$ am grateful to [name removed for blind review] for technical discussion concerning the use of truth predicates and countable transitive models.

Proof. (Sketch) Begin by proving the Reflection Theorem for $\mathbf{Z F C} \mathbf{C}_{T r}$. Then, reflect on the formula " $\phi$ ' is a Gödel code of a formula and $\operatorname{Tr}\left({ }^{r} \phi{ }^{\urcorner}\right)$" to obtain a $V_{\alpha}$ elementarily equivalent to $V$ (since for a given natural number $\left.\left.n={ }^{\ulcorner} \phi\right\urcorner, V \models \operatorname{Tr}\left({ }^{\ulcorner} \phi\right\urcorner\right)$ iff $V_{\alpha} \models \operatorname{Tr}\left({ }^{\ulcorner} \phi^{\urcorner}\right)$, and hence $V \models \phi$ iff $\left.V_{\alpha} \models \phi\right)$. Then Skolemise and Collapse over $V_{\alpha}$ to get the desired countable transtive model.
} 
we obtain a countable transitive model elementarily equivalent to $V$. This argument, in combination with the informal use of the Löwenheim-Skolem Theorem, shows that the existence of a countable transitive model elementarily equivalent to $V$ is little ontological price to pay, if any.

\section{Conclusion}

We have seen that Universist has some reason to want to use forcing to prove theorems and formulate axioms about $V$. Whilst the use of a Boolean ultrapower sometimes results in non-well-founded models, it suggests a class of forcings of special interest for the Universist; those for which the Boolean ultrapower can be kept wellfounded, and thus she has a particularly natural interpretation of forcing. The countable transitive model strategy, augmented to yield a model elementarily equivalent to $V$, we found to be an effective, flexible, and well-motivated method for interpreting forcing constructions. The extent to which one thinks that Hamkins' complaints have dialectic force against the Universist, thus turns precisely on whether or not one thinks that the Universist must incorporate actually uncountable sets in her interpretation of forcing. Perhaps such an argument can be made, but for now the Universist should remain largely unfazed by criticisms stemming from forcing constructions.

\section{References}

[Antos et al., S] Antos, C., Barton, N., and Friedman, S.-D. (S). Universism and extensions of $V$. Submitted.

[Antos and Friedman, F] Antos, C. and Friedman, S.-D. (F). Hyperclass forcing in Morse-Kelley class theory. Forthcoming in Journal of Symbolic Logic.

[Antos et al., 2015] Antos, C., Friedman, S.-D., Honzik, R., and Ternullo, C. (2015). Multiverse conceptions in set theory. Synthese, 192(8):2463-2488.

[Arrigoni and Friedman, 2013] Arrigoni, T. and Friedman, S.-D. (2013). The Hyperuniverse Program. Bulletin of Symbolic Logic, 19:77-96.

[Baumgartner and Hajnal, 1973] Baumgartner, J. and Hajnal, A. (1973). A proof (involving Martin's Axiom) of a partition relation. Fundamenta Mathematicae, 78(3):193-203.

[Cohen, 1966] Cohen, P. (1966). Set Theory and The Continuum Hypothesis. W.A. Benjamin, Inc.

[Cummings, 2010] Cummings, J. (2010). Iterated Forcing and Elementary Embeddings, pages 775-883. Springer Netherlands, Dordrecht.

[Feferman, 1969] Feferman, S. (1969). Set-theoretical foundations of category theory. In Reports of the Midwest Category Seminar III, Lecture Notes in Mathematics Volume 106, pages 201-247. Springer.

[Foreman, 1986] Foreman, M. (1986). Potent axioms. Transactions of the American Mathematical Society, 294(1):1-28.

[Foreman, 1998] Foreman, M. (1998). Generic large cardinals: New axioms for mathematics? Documenta Mathematica, 2:11-21. 
[Foreman, 2010] Foreman, M. (2010). Ideals and generic elementary embeddings. In Kanamori, A. and Foreman, M., editors, Handbook of Set Theory, pages 885-1147. Springer.

[Friedman, 2000] Friedman, S.-D. (2000). Fine Structure and Class Forcing. de Gruyter. de Gruyter Series in Logic and its Applications, Vol. 3.

[Friedman, 2006] Friedman, S.-D. (2006). Internal consistency and the inner model hypothesis. Bulletin of Symbolic Logic, 12(4):591-600.

[Friedman, 2010] Friedman, S.-D. (2010). Constructibility and class forcing. In Kanamori, A., editor, Handbook of Set Theory, pages 557-604. Springer.

[Friedman, F] Friedman, S.-D. (F). Evidence for set-theoretic truth and the hyperuniverse programme. IFCoLog Journal of Logic and its Applications. Forthcoming.

[Gitman et al., 2017] Gitman, V., Hamkins, J. D., Holy, P., Schlicht, P., and Williams, K. (2017). The exact strength of the class forcing theorem.

[Hamkins, 2003] Hamkins, J. D. (2003). A simple maximality principle. The Journal of Symbolic Logic, 68(2):527-550.

[Hamkins, 2012] Hamkins, J. D. (2012). The set-theoretic multiverse. The Review of Symbolic Logic, 5(3):416-449.

[Hamkins and Seabold, 2012] Hamkins, J. D. and Seabold, D. E. (2012). Wellfounded Boolean ultrapowers as large cardinal embeddings. arXiv:1206.6075 [math.LO].

[Hamkins and Yang, 2013] Hamkins, J. D. and Yang, R. (2013). Satisfaction is not absolute. arXiv:1312.0670v1 [math.LO].

[Holy et al., 2016] Holy, P., Krapf, R., Lücke, P., Njegomir, A., and Schlicht, P. (2016). Class forcing, the forcing theorem and boolean completions. The Journal of Symbolic Logic, 81(4):1500-1530.

[Holy et al., 2017] Holy, P., Krapf, R., and Schlicht, P. (2017). Characterizations of pretameness and the ord-cc.

[Jech, 2002] Jech, T. (2002). Set Theory. Springer.

[Jensen, 1972] Jensen, R. (1972). The fine structure of the constructible hierarchy. Annals of Mathematical Logic, 4:229-308.

[Kanamori, 2009] Kanamori, A. (2009). The Higher Infinite: Large Cardinals in Set Theory from Their Beginnings. Springer, 2nd edition.

[Koellner, 2013] Koellner, P. (2013). Hamkins on the multiverse. In Koellner, P., editor, Exploring the Frontiers of Incompleteness.

[Koellner, 2014] Koellner, P. (2014). Large cardinals and determinacy. In Zalta, E. N., editor, The Stanford Encyclopedia of Philosophy. Metaphysics Research Lab, Stanford University, spring 2014 edition.

[Kunen, 1971] Kunen, K. (1971). Elementary embeddings and infinitary combinatorics. The Journal of Symbolic Logic, 36:407-413.

[Kunen, 2013] Kunen, K. (2013). Set Theory. College Publications. 
[Larson, 2004] Larson, P. (2004). The Stationary Tower: Notes on a Course by W. Hugh Woodin. University lecture series. American Mathematical Society.

[Malliaris and Shelah, 2016] Malliaris, M. and Shelah, S. (2016). Cofinality spectrum theorems in model theory, set theory, and general topology. Journal of the American Mathematical Society, (29):237-297.

[Painlevé, 1900] Painlevé, P. (1900). Analyse des travaux scientifiques. Gauthier-Villars.

[Schindler, 2000] Schindler, R. (2000). Proper forcing and remarkable cardinals: I. Bulletin of Symbolic Logic, 6(2):176-184.

[Schindler, 2001] Schindler, R. (2001). Proper forcing and remarkable cardinals: II. Journal of Symbolic Logc, 66(3):1481-1492.

[Schindler, 2014] Schindler, R. (2014). Set Theory: Exploring Independence and Truth. Springer.

[Shoenfield, 1967] Shoenfield, J. (1967). Mathematical Logic. Addison-Wesley Publishing Co.

[Smullyan and Fitting, 1996] Smullyan, R. M. and Fitting, M. (1996). Set Theory and the Continuum Problem. Clarendon Press.

[Steel, 1996] Steel, J. (1996). The core model iterability problem. In Lecture Notes in Logic, volume 8. Springer.

[Todorčević and Farah, 1995] Todorčević, S. and Farah, I. (1995). Some Applications of the Method of Forcing. Yenisei, Moscow. 